\title{
A plactic algebra of extremal weight crystals and the Cauchy identity for Schur operators
}

\author{
Jae-Hoon Kwon
}

Received: 6 July 2010 / Accepted: 27 January 2011 / Published online: 18 February 2011

(C) Springer Science+Business Media, LLC 2011

\begin{abstract}
We give a new bijective interpretation of the Cauchy identity for Schur operators which is a commutation relation between two formal power series with operator coefficients. We introduce a plactic algebra associated with the Kashiwara's extremal weight crystals over the Kac-Moody algebra of type $A_{+\infty}$, and construct a Knuth type correspondence preserving the plactic relations. This bijection yields the Cauchy identity for Schur operators as a homomorphic image of its associated identity for plactic characters of extremal weight crystals, and also recovers Sagan and Stanley's correspondence for skew tableaux as its restriction.
\end{abstract}

Keywords Plactic algebra $\cdot$ Crystal $\cdot$ Schur operator

\section{Introduction}

Let $\Lambda=\Lambda_{\mathbf{x}}$ be the algebra of symmetric functions in a set of formal commuting variables $\mathbf{x}=\left\{x_{1}, x_{2}, \ldots\right\}$ over $\mathbb{Q}$. We denote by $\mathcal{P}$ the set of partitions and let $s_{\lambda}(\mathbf{x})$ be the Schur function in $\mathbf{x}$ corresponding to $\lambda \in \mathscr{P}$. Let

$$
\mathcal{P}(\mathbf{x})=\sum_{\lambda \in \mathcal{P}} s_{\lambda} s_{\lambda}(\mathbf{x}), \quad \mathcal{Q}(\mathbf{x})=\sum_{\lambda \in \mathcal{P}} s_{\lambda}^{\perp} s_{\lambda}(\mathbf{x}) \in \operatorname{End}_{\mathbb{Q}}(\Lambda)[[\mathbf{x}]],
$$

where $s_{\lambda}$ and $s_{\lambda}^{\perp}$ are linear operators on $\Lambda$ induced from the left multiplication by $s_{\lambda}(\mathbf{x})$ and its adjoint with respect to the Hall inner product on $\Lambda$, respectively. One may regard $s_{\lambda}$ and $s_{\lambda}^{\perp}$ as operators on $\mathbb{Q} \mathcal{P}=\bigoplus_{\lambda \in \mathcal{P}} \mathbb{Q} \lambda$, where $\lambda$ is identified with

This work was supported by KRF Grant 2008-314-C00004.

J.-H. Kwon (西)

Department of Mathematics, University of Seoul, Seoul 130-743, Korea

e-mail: jhkwon@uos.ac.kr 
$s_{\lambda}(\mathbf{x})$. Moreover $s_{\lambda}$ and $s_{\lambda}^{\perp}$ can be given as Schur functions in certain locally noncommutative operators on $\mathbb{Q} \mathcal{P}$ called Schur operators by Fomin, while $\mathcal{P}(\mathbf{x})$ and $\mathcal{Q}(\mathbf{x})$ can be written as Cauchy products in Schur operators and $\mathbf{x}[3,4]$.

Let $\mathbf{y}=\left\{y_{1}, y_{2}, \ldots\right\}$ be another set of formal commuting variables. It is well known that the following commutation relation holds:

$$
\mathcal{Q}(\mathbf{y}) \mathcal{P}(\mathbf{x})=\frac{1}{\prod_{i, j}\left(1-x_{i} y_{j}\right)} \mathcal{P}(\mathbf{x}) \mathcal{Q}(\mathbf{y})
$$

called generalized Cauchy identity or Cauchy identity for Schur operators. Considering both sides as operators with coefficients in $\Lambda_{\mathbf{x}} \otimes \Lambda_{\mathbf{y}}$ and then equating each entry of their matrix forms, we obtain a Cauchy identity for skew Schur functions [16],

$$
\sum_{\lambda} s_{\lambda / \alpha}(\mathbf{x}) s_{\lambda / \beta}(\mathbf{y})=\frac{1}{\prod_{i, j}\left(1-x_{i} y_{j}\right)} \sum_{\eta} s_{\beta / \eta}(\mathbf{x}) s_{\alpha / \eta}(\mathbf{y}),
$$

where $\alpha, \beta$ are given partitions. A bijective interpretation of the Cauchy identity for skew Schur functions was given by Sagan and Stanley [17], and it was extended to a bijection in a more general framework by Fomin [3] including various analogues of Knuth correspondence.

Recently, a new representation theoretic interpretation of the Cauchy identity for Schur operators was given by the author [11] using the notion of Kashiwara's extremal weight crystals [8] over the quantized enveloping algebra associated with the Kac-Moody algebra of type $A_{+\infty}$, say $\mathfrak{g l}_{>0}$. It is proved that a Schur operator can be realized as a functor of tensoring by an extremal weight crystal element and (1.1) can be understood as a non-commutative character identity corresponding to the decomposition of the crystal graph of the Fock space with infinite positive level, which is an infinite analogue of the level $n$ fermionic Fock space decomposition due to Frenkel [5].

Motivated by a categorification of Schur operators in [11], we give a new combinatorial way to explain both the Cauchy identities for Schur operators and skew Schur functions in terms of a single bijection. More precisely, the main result in this paper is to construct a Knuth type correspondence, which gives a bijective interpretation of the identity (1.1) or its dual form, as the usual Knuth correspondence does for the Cauchy product, and also recovers the Sagan and Stanley's correspondence as its restriction.

Our approach is to define a $t$-analogue of the plactic algebra $U(t)$ for $\mathfrak{g l}_{>0}$ generated by $u_{i}$ and $u_{i} \vee$ for $i \in \mathbb{N}$ with $t$ an indeterminate, where the subalgebra generated by $u_{i}$ (resp. $\left.u_{i} \vee\right)(i \in \mathbb{N}$ ) is isomorphic to the usual plactic algebra introduced by Lascoux and Schützenberger [14]. We show that $\mathcal{U}(1)$ is isomorphic to the plactic algebra defined by using the notion of crystal equivalence (cf. [15]). Note that each monomial in $\mathcal{U}(1)$ corresponds in general to an element of an extremal weight crystal, which may not be either highest weight or lowest weight crystal.

Now, let $\mathcal{M}_{\mathbb{A}, \mathbb{B}}$ be the set of $\mathbb{A} \times \mathbb{B}$ matrices $A=\left(a_{i j}\right)$ with entries in $\mathbb{Z}_{\geq 0}$ such that $\sum_{i \in \mathbb{A}} \sum_{j \in \mathbb{B}} a_{i j}<\infty$ and $a_{i j} \leq 1$ for $|i| \neq|j|$, where $\mathbb{A}$ and $\mathbb{B}$ are arbitrary $\mathbb{Z}_{2^{-}}$ graded sets and $|\cdot|$ denotes the degree of an element in $\mathbb{A}$ or $\mathbb{B}$. We assume that all the elements in $\mathbb{N}$ and $\mathbb{N}^{\vee}=\left\{i^{\vee} \mid i \in \mathbb{N}\right\}$ are of degree 0 . By using the usual Knuth 
map and non-commutative Littlewood-Richardson rule of extremal weight crystals for $\mathfrak{g l}_{>0}[11,12]$, we construct an explicit bijection (Theorem 5.1);

$$
\mathcal{M}_{\mathbb{A}, \mathbb{N}} \times \mathcal{M}_{\mathbb{B}, \mathbb{N}^{\vee}} \longrightarrow \mathcal{M}_{\mathbb{A}, \mathbb{B}} \times \mathcal{M}_{\mathbb{B}, \mathbb{N}^{\vee}} \times \mathcal{M}_{\mathbb{A}, \mathbb{N}},
$$

which preserves the weights with respect to $\mathbb{A}$ and $\mathbb{B}$, and the plactic relations of $\mathcal{U}(t)$ for the column words with entries in $\mathbb{N} \cup \mathbb{N}^{\vee}$ on both sides. As a corollary, we obtain a character identity in locally non-commuting variables $\mathbf{u}=\left\{u_{i}, u_{i} \vee \mid i \in \mathbb{N}\right\}$ and commuting variables $\mathbf{x}_{\mathbb{A}}=\left\{x_{a} \mid a \in \mathbb{A}\right\}, \mathbf{x}_{\mathbb{B}}=\left\{x_{b} \mid b \in \mathbb{B}\right\}$ (Corollary 5.2). In particular, when $\mathbb{A}=\mathbb{B}=\mathbb{N}$, this identity recovers (1.1) under a homomorphism sending $u_{i}$ and $u_{i} \vee$ to Schur operators on $\mathbb{Q} \mathcal{P}$ and specializing $t=1$. Moreover, the Knuth correspondence for skew tableaux by Sagan and Stanley can be recovered by restricting the above bijection to the pairs of matrices on the left-hand side whose column words are Littlewood-Richardson words of shape $(\alpha, \beta)$ with $\alpha, \beta \in \mathcal{P}$ (see Sect. 5.3 for a definition).

The paper is organized as follows. In Sect. 2, we briefly recall necessary background for semistandard tableaux and the Knuth correspondence. In Sect. 3, we recall the notion of rational semistandard tableaux for $\mathfrak{g l}_{>0}$ and their insertion algorithm. In Sect. 4 , we introduce a plactic algebra for $\mathfrak{g l}_{>0}$ associated with rational semistandard tableaux. Finally, in Sect. 5, we construct a Knuth type correspondence and its associated non-commutative character identity.

\section{Preliminaries}

\subsection{Semistandard tableaux}

Throughout this paper, we assume that $\mathbb{A}$ (or $\mathbb{B}$ ) is a linearly ordered $\mathbb{Z}_{2}$-graded set, that is, $\mathbb{A}=\mathbb{A}_{0} \sqcup \mathbb{A}_{1}$, which is at most countable. We usually denote by $<$ a linear ordering on a given linearly ordered $\mathbb{Z}_{2}$-graded set. For $a \in \mathbb{A}_{\epsilon}\left(\epsilon \in \mathbb{Z}_{2}\right)$, we put $|a|=\epsilon$. By convention, we let $\mathbb{N}=\{1<2<\cdots\}$, and $[n]=\{1<\cdots<n\}$ for $n \geq 1$, where all the elements are of degree 0 .

Let $\mathcal{P}$ denote the set of partitions. We identify a partition $\lambda=\left(\lambda_{i}\right)_{i \geq 1}$ with a Young diagram or a subset $\left\{(i, j) \mid 1 \leq j \leq \lambda_{i}\right\}$ of $\mathbb{N} \times \mathbb{N}$ following [16]. Let $\ell(\lambda)=$ $\left|\left\{i \mid \lambda_{i} \neq 0\right\}\right|$. We denote by $\lambda^{\prime}=\left(\lambda_{i}^{\prime}\right)_{i \geq 1}$ the conjugate partition of $\lambda$ whose Young diagram is $\{(i, j) \mid(j, i) \in \lambda\}$. For $\mu \in \mathcal{P}$ with $\lambda \supset \mu, \lambda / \mu$ denotes the skew Young diagram.

For a skew Young diagram $\lambda / \mu$, a tableau $T$ obtained by filling $\lambda / \mu$ with entries in $\mathbb{A}$ is called $\mathbb{A}$-semistandard if (1) the entries in each row (resp. column) are weakly increasing from left to right (resp. from top to bottom), (2) the entries in $\mathbb{A}_{0}$ (resp. $\mathbb{A}_{1}$ ) are strictly increasing in each column (resp. row). We say that $\lambda / \mu$ is the shape of $T$, and write $\operatorname{sh}(T)=\lambda / \mu$. We denote by $T(i, j)$ the entry of $T$ at $(i, j) \in \lambda / \mu$.

We denote by $S S T_{\mathbb{A}}(\lambda / \mu)$ the set of all $\mathbb{A}$-semistandard tableaux of shape $\lambda / \mu$. We set $\mathcal{P}_{\mathbb{A}}=\left\{\lambda \in \mathcal{P} \mid S S T_{\mathbb{A}}(\lambda) \neq \emptyset\right\}$. For example, $\mathcal{P}_{\mathbb{A}}=\mathcal{P}$ when $\mathbb{A}$ is an infinite set, and $\mathcal{P}_{[n]}=\{\lambda \mid \ell(\lambda) \leq n\}$.

Let $\mathcal{W}_{\mathbb{A}}$ be the set of finite words with letters in $\mathbb{A}$. For $T \in S S T_{\mathbb{A}}(\lambda / \mu)$, we denote by $w(T)=w_{\text {col }}(T) \in \mathcal{W}_{\mathbb{A}}$ the word obtained by reading the entries of $T$ column by column from right to left, and from top to bottom in each column. 
Let $P_{\mathbb{A}}=\bigoplus_{a \in \mathbb{A}} \mathbb{Z} \epsilon_{a}$ be the free abelian group with a basis $\left\{\epsilon_{a} \mid a \in \mathbb{A}\right\}$ and let $\mathbf{x}_{\mathbb{A}}=\left\{x_{a} \mid a \in \mathbb{A}\right\}$ be a set of formal commuting variables. For $\lambda=\sum_{a \in \mathbb{A}} \lambda_{a} \epsilon_{a} \in P_{\mathbb{A}}$, let $\mathbf{x}_{\mathbb{A}}^{\lambda}=\prod_{a \in \mathbb{A}} x_{a}^{\lambda_{a}}$. For $w=w_{1} \ldots w_{r} \in \mathcal{W}_{\mathbb{A}}$, we define $\mathrm{wt}_{\mathbb{A}}(w)=\sum_{1 \leq i \leq r} \epsilon_{w_{i}} \in$ $P_{\mathbb{A}}$. For $T \in S S T_{\mathbb{A}}(\lambda / \mu)$, we define $\operatorname{wt}_{\mathbb{A}}(T)=\sum_{(i, j) \in \lambda / \mu} \epsilon_{T(i, j)}$. Let $s_{\lambda / \mu}\left(\mathbf{x}_{\mathbb{A}}\right)=$ $\sum_{T \in S S T_{\mathbb{A}}(\lambda / \mu)} \mathbf{x}_{\mathbb{A}}^{\mathrm{wt}_{\mathbb{A}}(T)}$, which is the character of $\operatorname{SST}_{\mathbb{A}}(\lambda / \mu)$. Note that $s_{\lambda / \mu}\left(\mathbf{x}_{\mathbb{A}}\right)$ is a usual (skew) Schur function when $\mathbb{A}=\mathbb{N}$.

We will also use the following operations on tableaux.

(1) dual: Let $\mathbb{A}^{\vee}=\left\{a^{\vee} \mid a \in \mathbb{A}\right\}$ be the linearly ordered $\mathbb{Z}_{2}$-graded set with $\left|a^{\vee}\right|=|a|$ and $a_{1}^{\vee}<a_{2}^{\vee}$ for $a_{1}>a_{2}$. For $T \in S S T_{\mathbb{A}}(\lambda / \mu)$, we define $T^{\vee}$ to be the tableau obtained by applying $180^{\circ}$-rotation to $T$ and replacing each entry $a$ in $T$ with $a^{\vee}$. Then $T^{\vee} \in S S T_{\mathbb{A}^{\vee}}\left((\lambda / \mu)^{\vee}\right)$, where $(\lambda / \mu)^{\vee}$ denotes the shape of $T^{\vee}$. We use the convention that $\left(a^{\vee}\right)^{\vee}=a$ for $a \in \mathbb{A}$ and hence $\left(T^{\vee}\right)^{\vee}=T$.

(2) gluing: Let $\mathbb{A} * \mathbb{B}$ be the $\mathbb{Z}_{2}$-graded set $\mathbb{A} \sqcup \mathbb{B}$ with the extended linear ordering given by $a<b$ for $a \in \mathbb{A}$ and $b \in \mathbb{B}$. For $S \in S S T_{\mathbb{A}}(\mu)$ and $T \in S S T_{\mathbb{B}}(\lambda / \mu)$, we define $S * T \in S S T_{\mathbb{A} * \mathbb{B}}(\lambda)$ by $S * T(i, j)=S(i, j)$ for $(i, j) \in \mu$ and $T(i, j)$ for $(i, j) \in \lambda / \mu$.

\subsection{Littlewood-Richardson rule}

For $a \in \mathbb{A}$ and $T \in S S T_{\mathbb{A}}(\lambda)$ with $\lambda \in \mathcal{P}_{\mathbb{A}}, a \rightarrow T$ (resp. $T \leftarrow a$ ) denotes the tableau obtained by the Schensted column (resp. row) insertion (see for example, [6, Appendix A.2] and [2] for its super-analogue). For $w=w_{1} \ldots w_{r} \in \mathcal{W}_{\mathbb{A}}$, we let $(w \rightarrow T)=\left(w_{r} \rightarrow\left(\cdots\left(w_{1} \rightarrow T\right) \cdots\right)\right)$. For $S \in S S T_{\mathbb{A}}(\mu)$ and $T \in S S T_{\mathbb{A}}(\nu)$ with $\mu, v \in \mathcal{P}_{\mathbb{A}}$, we define $(T \rightarrow S)=(w(T) \rightarrow S)$.

For $\lambda, \mu, \nu \in \mathcal{P}$ with $|\lambda|=|\mu|+|\nu|$, let $\mathbf{L R}_{\mu \nu}^{\lambda}$ be the set of tableaux $U$ in $S S T_{\mathbb{N}}(\lambda / \mu)$ such that

(1) $\operatorname{wt}_{\mathbb{N}}(U)=\sum_{i>1} v_{i} \epsilon_{i}$,

(2) for $1 \leq k \leq|v|$, the number of occurrences of each $i \geq 1$ in $w_{1} \ldots w_{k}$ is no less than that of $i+1$ in $w_{1} \ldots w_{k}$, where $w(U)=w_{1} \ldots w_{|v|}$.

We call $\mathbf{L} \mathbf{R}_{\mu \nu}^{\lambda}$ the set of Littlewood-Richardson tableaux of shape $\lambda / \mu$ with content $\nu$ and put $c_{\mu \nu}^{\lambda}=\left|\mathbf{L} \mathbf{R}_{\mu \nu}^{\lambda}\right|$ [16]. Let us introduce a variation of $\mathbf{L} \mathbf{R}_{\mu \nu}^{\lambda}$, which is necessary for our later arguments. We define $\overline{\mathbf{L R}}_{\mu \nu}^{\lambda}$ to be the set of tableaux $U$ in $\operatorname{SST}-\mathbb{N}(\lambda / \mu)$ such that

(1) wt $_{-\mathbb{N}}(U)=\sum_{i>1} v_{i} \epsilon_{-i}$,

(2) for $1 \leq k \leq|v|$, the number of occurrences of each $-i \leq-1$ in $w_{k} \ldots w_{|v|}$ is no less than that of $-(i+1)$ in $w_{k} \ldots w_{|v|}$, where $w(U)=w_{1} \ldots w_{|v|}$.

Note that for $U \in S S T_{\mathbb{N}}(\lambda / \mu), U \in \mathbf{L} \mathbf{R}_{\mu \nu}^{\lambda}$ if and only if $U$ is Knuth equivalent to $H_{v} \in S S T_{\mathbb{N}}(v)$, where $H_{v}(i, j)=i$ for $(i, j) \in v$ (cf. [6]). Similarly, we have for $U \in S S T_{-\mathbb{N}}(\lambda / \mu), U \in \overline{\mathbf{L R}}_{\mu \nu}^{\lambda}$ if and only if $U$ is Knuth equivalent to $L_{v} \in S S T_{-\mathbb{N}}(v)$, where $L_{v}(i, j)=-v_{j}^{\prime}+i-1$ for $(i, j) \in v$.

There is also a one-to-one correspondence from the set of $V \in S S T_{\mathbb{N}}(v)$ such that $\left(V \rightarrow H_{\mu}\right)=H_{\lambda}$ to $\mathbf{L} \mathbf{R}_{\mu \nu}^{\lambda}$. Indeed, $V$ corresponds to $l(V) \in \mathbf{L} \mathbf{R}_{\mu \nu}^{\lambda}$ where the number of $k$ 's in the $i$ th row of $V$ is equal to the number of $i$ 's in the $k$ th row of $l(V)$ for $i, k \geq 1$. 
Example 2.1

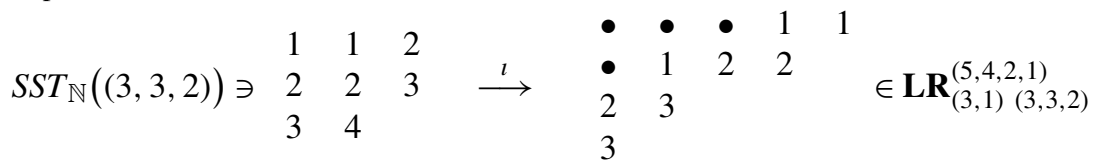

For $S \in S S T_{\mathbb{A}}(\mu)$ and $T \in S S T_{\mathbb{A}}(v)$ with $\mu, v \in \mathcal{P}_{\mathbb{A}}$, suppose that $\lambda=\operatorname{sh}(T \rightarrow S)$ and $w(T)=w_{1} \ldots w_{r}$. Define $(T \rightarrow S)_{R}$ to be the tableau of shape $\lambda / \mu$ such that $\operatorname{sh}\left(w_{1} \ldots w_{k} \rightarrow S\right) / \operatorname{sh}\left(w_{1} \ldots w_{k-1} \rightarrow S\right)$ is filled with $i$ when $w_{k}$ appears in the $i$ th row of $T$ for $1 \leq k \leq r$. Then the map $(S, T) \mapsto\left((T \rightarrow S),(T \rightarrow S)_{R}\right)$ gives a bijection [20]

$$
S S T_{\mathbb{A}}(\mu) \times S S T_{\mathbb{A}}(\nu) \longrightarrow \bigsqcup_{\lambda \in \mathcal{P}_{\mathbb{A}}} S S T_{\mathbb{A}}(\lambda) \times \mathbf{L} \mathbf{R}_{\mu \nu}^{\lambda},
$$

which also implies $s_{\mu}\left(\mathbf{x}_{\mathbb{A}}\right) s_{\nu}\left(\mathbf{x}_{\mathbb{A}}\right)=\sum_{\lambda} c_{\mu \nu}^{\lambda} s_{\lambda}\left(\mathbf{x}_{\mathbb{A}}\right)$.

\subsection{Skew Littlewood-Richardson rule}

Let $\lambda / \mu$ be a skew Young diagram. Let $U$ be a tableau of shape $\lambda / \mu$ with entries in $\mathbb{A} \sqcup \mathbb{B}$, satisfying the following conditions;

(S1) $U(i, j) \leq U\left(i^{\prime}, j^{\prime}\right)$ whenever $U(i, j), U\left(i^{\prime}, j^{\prime}\right) \in \mathbb{X}$ for $(i, j),\left(i^{\prime}, j^{\prime}\right) \in \lambda / \mu$ with $i \leq i^{\prime}$ and $j \leq j^{\prime}$,

(S2) in each column of $U$, entries in $\mathbb{X}_{0}$ increase strictly from top to bottom,

(S3) in each row of $U$, entries in $\mathbb{X}_{1}$ increase strictly from left to right,

where $\mathbb{X}=\mathbb{A}$ or $\mathbb{B}$. Suppose that $a \in \mathbb{A}$ and $b \in \mathbb{B}$ are two adjacent entries in $U$ such that $b$ is placed above or to the left of $a$. Interchanging $a$ and $b$ is called a switching if the resulting tableau still satisfies the conditions (S1), (S2) and (S3).

For $T \in S S T_{\mathbb{A}}(\lambda / \mu)$, let $U$ be a tableau obtained from $H_{\mu} * T$ by applying switching procedures as far as possible (in this case, $\mathbb{B}=\mathbb{N}$ ). Then $U=J(T) * J(T)_{R}$ for some $J(T) \in S S T_{\mathbb{A}}(\nu)$ and $J(T)_{R} \in S S T_{\mathbb{N}}(\lambda / \nu)$ with $v \in \mathcal{P}_{\mathbb{A}}$. Then by [1, Theorem 3.1], the map sending $T$ to $\left(J(T), J(T)_{R}\right)$ gives a bijection

$$
S S T_{\mathbb{A}}(\lambda / \mu) \longrightarrow \bigsqcup_{\nu \in \mathcal{P}_{\mathbb{A}}} S S T_{\mathbb{A}}(\nu) \times \mathbf{L} \mathbf{R}_{\nu \mu}^{\lambda}
$$

In particular, the map $Q \mapsto J(Q)_{R}$ restricts to a bijection from $\mathbf{L} \mathbf{R}_{\mu \nu}^{\lambda}$ to $\mathbf{L} \mathbf{R}_{v \mu}^{\lambda}$, and from $\overline{\mathbf{L R}}_{\mu \nu}^{\lambda}$ to $\mathbf{L} \mathbf{R}_{\nu \mu}^{\lambda}$ when $\mathbb{A}= \pm \mathbb{N}$, respectively.

\subsection{Knuth correspondence}

Let $\mathcal{M}_{\mathbb{A}, \mathbb{B}}$ be the set of $\mathbb{A} \times \mathbb{B}$ matrices $A=\left(a_{i j}\right)$ with entries in $\mathbb{Z}_{\geq 0}$ such that $\sum_{i \in \mathbb{A}} \sum_{j \in \mathbb{B}} a_{i j}<\infty$ and $a_{i j} \leq 1$ for $|i| \neq|j|$. Let $\Omega_{\mathbb{A}, \mathbb{B}}$ be the set of biwords $(\mathbf{i}, \mathbf{j}) \in$ $\mathcal{W}_{\mathbb{A}} \times \mathcal{W}_{\mathbb{B}}$ such that 
(1) $\mathbf{i}=i_{1} \cdots i_{r}$ and $\mathbf{j}=j_{1} \cdots j_{r}$ for some $r \geq 0$,

(2) $\left(i_{1}, j_{1}\right) \leq \cdots \leq\left(i_{r}, j_{r}\right)$,

(3) $\left(i_{s}, j_{s}\right)<\left(i_{s+1}, j_{s+1}\right)$ if $\left|i_{s}\right| \neq\left|j_{s}\right|$ for $1 \leq s<r$,

where for $(i, j)$ and $(k, l) \in \mathbb{A} \times \mathbb{B}$,

$$
(i, j)<(k, l) \Longleftrightarrow \begin{cases}(i<k) & \text { or, } \\ (i=k,|i|=0, \text { and } j>l) & \text { or, } \\ (i=k,|i|=1, \text { and } j<l) . & \end{cases}
$$

There is a bijection from $\Omega_{\mathbb{A}, \mathbb{B}}$ to $\mathcal{M}_{\mathbb{A}, \mathbb{B}}$, where $(\mathbf{i}, \mathbf{j})$ is mapped to $A(\mathbf{i}, \mathbf{j})=\left(a_{i j}\right)$ with $a_{i j}=\left|\left\{k \mid\left(i_{k}, j_{k}\right)=(i, j)\right\}\right|$. Note that the pair of empty words $(\emptyset, \emptyset)$ corresponds to zero matrix.

For $A=A(\mathbf{i}, \mathbf{j}) \in \mathcal{M}_{\mathbb{A}, \mathbb{B}}$, we let $P(A)=(\mathbf{j} \rightarrow \emptyset)$, where $\emptyset$ is the empty tableau, and let $Q(A)$ be the tableau of the same shape as $P(A)$ such that $\operatorname{sh}\left(j_{1} \ldots j_{k} \rightarrow\right.$ $\emptyset) / \operatorname{sh}\left(j_{1} \ldots j_{k-1} \rightarrow \emptyset\right)$ is filled with $i_{k}$ for $k \geq 1$. Then the map sending $A$ to $(P(A), Q(A))$ gives a bijection

$$
\mathcal{M}_{\mathbb{A}, \mathbb{B}} \longrightarrow \bigsqcup_{\lambda \in \mathcal{P}_{\mathbb{A}} \cap \mathcal{P}_{\mathbb{B}}} S S T_{\mathbb{B}}(\lambda) \times S S T_{\mathbb{A}}(\lambda),
$$

which is the (super-analogue of) Knuth (or RSK) correspondence [10]. If we define $\mathrm{wt}_{\mathbb{B}}(A)=\mathrm{wt}_{\mathbb{B}}(\mathbf{j})$ and $\mathrm{wt}_{\mathbb{A}}(A)=\mathrm{wt}_{\mathbb{A}}(\mathbf{i})$, then the bijection preserves $\mathrm{wt}_{\mathbb{A}}$ and $\mathrm{wt}_{\mathbb{B}}$. In terms of characters, we obtain the following Cauchy identity:

$$
\frac{\prod_{|a| \neq|b|}\left(1+x_{a} x_{b}\right)}{\prod_{|a|=|b|}\left(1-x_{a} x_{b}\right)}=\sum_{\lambda \in \mathcal{P}_{\mathbb{A}} \cap \mathcal{P}_{\mathbb{B}}} s_{\lambda}\left(\mathbf{x}_{\mathbb{B}}\right) s_{\lambda}\left(\mathbf{x}_{\mathbb{A}}\right),
$$

where $a \in \mathbb{A}$ and $b \in \mathbb{B}$.

Similarly, for $A=\left(a_{i j}\right) \in \mathcal{M}_{\mathbb{A}, \mathbb{B}}$, let $A^{\prime}=\left(a_{i j^{\vee}}^{\prime}\right)$ be the unique matrix in $\mathcal{M}_{\mathbb{A}, \mathbb{B}^{\vee}}$ such that $a_{i j}=a_{i j \vee}^{\prime}$ for $(i, j) \in \mathbb{A} \times \mathbb{B}$. Then the map sending $A$ to $\left(P\left(A^{\prime}\right)^{\vee}, Q\left(A^{\prime}\right)\right)$ gives a bijection

$$
\mathcal{M}_{\mathbb{A}, \mathbb{B}} \longrightarrow \bigsqcup_{\lambda \in \mathcal{P}_{\mathbb{A}} \cap \mathcal{P}_{\mathbb{B}}} S S T_{\mathbb{B}}\left(\lambda^{\vee}\right) \times S S T_{\mathbb{A}}(\lambda) .
$$

Finally, for $\mu \in \mathcal{P}_{\mathbb{A}}$, we have

$$
\begin{aligned}
& S S T_{\mathbb{A}}(\mu) \times \mathcal{M}_{\mathbb{A}, \mathbb{B}} \\
& \stackrel{1-1}{\longrightarrow} \bigsqcup_{\nu \in \mathcal{P}_{\mathbb{A}} \cap \mathcal{P}_{\mathbb{B}}} S S T_{\mathbb{A}}(\mu) \times S S T_{\mathbb{B}}(\nu) \times S S T_{\mathbb{A}}(\nu) \quad \text { by (2.3) } \\
& \stackrel{1-1}{\longleftrightarrow} \bigsqcup_{\substack{\lambda \in \mathcal{P}_{\mathbb{A}} \\
\mu \subset \lambda}} S S T_{\mathbb{A}}(\lambda) \times\left(\bigsqcup_{\nu \in \mathcal{P}_{\mathbb{B}}} S S T_{\mathbb{B}}(\nu) \times \mathbf{L} \mathbf{R}_{v \mu}^{\lambda}\right) \quad \text { by (2.1) } \\
& \stackrel{1-1}{\longleftrightarrow} \bigsqcup_{\substack{\lambda \in \mathcal{P}_{\mathbb{A}} \\
\mu \subset \lambda}} S S T_{\mathbb{A}}(\lambda) \times S S T_{\mathbb{B}}(\lambda / \mu) \quad \text { by (2.2) }
\end{aligned}
$$


Hence we obtain a bijection

$$
S S T_{\mathbb{A}}(\mu) \times \mathcal{M}_{\mathbb{A}, \mathbb{B}} \longrightarrow \bigsqcup_{\substack{\lambda \in \mathcal{P}_{\mathbb{A}} \\ \mu \subset \lambda}} S S T_{\mathbb{A}}(\lambda) \times S S T_{\mathbb{B}}(\lambda / \mu)
$$

Also by using (2.4), we have a bijection

$$
S S T_{\mathbb{A}}(\mu) \times \mathcal{M}_{\mathbb{A}, \mathbb{B}} \longrightarrow \bigsqcup_{\substack{\lambda \in \mathcal{P}_{\mathbb{A}} \\ \mu \subset \lambda}} S S T_{\mathbb{A}}(\lambda) \times S S T_{\mathbb{B}}\left((\lambda / \mu)^{\vee}\right)
$$

\section{Rational semistandard tableaux}

3.1 Rational semistandard tableaux for $\mathfrak{g l}_{>0}$

For convenience, we let for a skew Young diagram $\lambda / \mu$,

$$
\mathcal{B}_{\lambda / \mu}=S S T_{\mathbb{N}}(\lambda / \mu), \quad \mathcal{B}_{\lambda / \mu}^{\vee}=S S T_{\mathbb{N}^{\vee}}\left((\lambda / \mu)^{\vee}\right) .
$$

Definition 3.1 For $\mu, v \in \mathcal{P}$, we define $\mathcal{B}_{\mu, v}$ to be the set of bitableaux $(S, T)$ such that

(1) $(S, T) \in \mathcal{B}_{\mu} \times \mathcal{B}_{\nu}^{\vee}$,

(2) $|\{i \mid S(i, 1) \leq k\}|+\left|\left\{i \mid T^{\vee}(i, 1) \leq k\right\}\right| \leq k$ for $k \geq 1$.

For convenience, we identify $\mathcal{B}_{\mu, \emptyset}$ and $\mathcal{B}_{\emptyset, v}$ with $\mathcal{B}_{\mu}$ and $\mathcal{B}_{v}^{\vee}$, respectively.

\section{Example 3.2}

$$
\left(\begin{array}{lllll}
1 & 1 & 3 & & 7^{\vee} \\
2 & 3 & & 5^{\vee} & 5^{\vee} \\
4 & & & 4^{\vee} & 3^{\vee}
\end{array}\right) \in \mathcal{B}_{(3,2,1),(2,2,1)}
$$

Remark 3.3 Let $\mathfrak{g l}_{>0}$ be the general linear Lie algebra spanned by $\mathbb{N} \times \mathbb{N}$ complex matrices of finite support. Then $\mathcal{B}_{\mu, \nu}$ parameterizes a basis of an extremal weight module over the quantized universal enveloping algebra $U_{q}\left(\mathfrak{g l}_{>0}\right)$ [11]. Recall that $\mathcal{B}_{\mu, \nu} \cap\left(S S T_{[n]}(\mu) \times S S T_{[n]^{\vee}}\left(v^{\vee}\right)\right)(n \geq 2)$ is a set of rational tableaux for $\mathfrak{g l}_{n}$ introduced by Stembridge, which parameterizes a basis of a finite dimensional complex irreducible representation of $\mathfrak{g l}_{n}$ [18].

Let us review an insertion algorithm for $\mathcal{B}_{\mu, \nu}[11]$, which is an infinite analogue of those for rational semistandard tableaux for $\mathfrak{g l}_{n}[18,19]$. For $a \in \mathbb{N}$ and $(S, T) \in \mathcal{B}_{\mu, \nu}$, we define $a \rightarrow(S, T)$ in the following way.

Suppose first that $S$ is the empty tableau and $T$ is a single column tableau. Let $\left(T^{\prime}, a^{\prime}\right)$ be the pair obtained as follows. 
(1) If $T$ contains $a^{\vee},(a+1)^{\vee}, \ldots,(b-1)^{\vee}$ as its entries but not $b^{\vee}$, then $T^{\prime}$ is the tableau obtained from $T$ by replacing $a^{\vee},(a+1)^{\vee}, \ldots,(b-1)^{\vee}$ with $(a+$ $1)^{\vee},(a+2)^{\vee}, \ldots, b^{\vee}$, and put $a^{\prime}=b$.

(2) If $T$ does not contain $a^{\vee}$, then leave $T$ unchanged and put $a^{\prime}=a$.

Now, we suppose that $S$ and $T$ are arbitrary.

(1) Apply the above process to the leftmost column of $T$ with $a$.

(2) Repeat (1) with $a^{\prime}$ and the next column to the right.

(3) Continue this process to the rightmost column of $T$ to get a tableau $T^{\prime}$ and $a^{\prime \prime}$.

(4) Define

$$
(a \rightarrow(S, T))=\left(\left(a^{\prime \prime} \rightarrow S\right), T^{\prime}\right) .
$$

Then $a \rightarrow(S, T) \in \mathcal{B}_{\sigma, v}$ for some $\sigma \in \mathcal{P}$ with $|\sigma / \mu|=1$. For $w=w_{1} \ldots w_{r} \in \mathcal{W}_{\mathbb{N}}$, we let $(w \rightarrow(S, T))=\left(w_{r} \rightarrow\left(\cdots\left(w_{1} \rightarrow(S, T)\right) \cdots\right)\right)$.

Next, we define $(S, T) \leftarrow a^{\vee}$ to be the pair $\left(S^{\prime}, T^{\prime}\right)$ obtained in the following way:

(1) If the pair $\left(S,\left(T^{\vee} \leftarrow a\right)^{\vee}\right)$ satisfies the condition (2) in Definition 3.1, then put $S^{\prime}=S$ and $T^{\prime}=\left(T^{\vee} \leftarrow a\right)^{\vee}$.

(2) Otherwise, choose the smallest $k$ such that $a_{k}$ is bumped out of the $k$ th row in the row insertion of $a$ into $T^{\vee}$ and the insertion of $a_{k}$ into the $(k+1)$-st row violates the condition (2) in Definition 3.1.

(2-a) Stop the row insertion of $a$ into $T^{\vee}$ when $a_{k}$ is bumped out, and let $T^{\prime}$ be the resulting tableau after taking $\vee$.

(2-b) Remove $a_{k}$ in the leftmost column of $S$, which necessarily exists, and then play the jeu de taquin (see for example [6, Sect. 1.2]) to obtain a tableau $S^{\prime}$.

In this case, $(S, T) \leftarrow a^{\vee} \in \mathcal{B}_{\sigma, \tau}$, where either (1) $|\mu / \sigma|=1$ and $\tau=\nu$, or (2) $\sigma=\mu$ and $|\tau / \nu|=1$. For $w=w_{1} \ldots w_{r} \in \mathcal{W}_{\mathbb{N} \vee}$, we let $((S, T) \leftarrow w)=((\cdots((S, T) \leftarrow$ $\left.\left.\left.w_{1}\right) \cdots\right) \leftarrow w_{r}\right)$.

\subsection{Non-commutative Littlewood-Richardson rule}

Let us recall the Littlewood-Richardson rule for $\mathcal{B}_{\mu, v}$ (see [11, Proposition 4.9] for more details).

Let $\mu, v \in \mathcal{P}$ be given. For $(S, T) \in \mathcal{B}_{v}^{\vee} \times \mathcal{B}_{\mu}$, consider $(w(T) \rightarrow(\emptyset, S))$. Suppose that $w(T)=w_{1} \ldots w_{r}$ and $\left(w_{1} \ldots w_{k} \rightarrow(\emptyset, S)\right) \in \mathcal{B}_{\mu^{(k)}, v}$ for $1 \leq k \leq r$. Let $\left(i_{k}, j_{k}\right)$ $\in \mu$ correspond to $w_{k}$ in $T(1 \leq k \leq r)$. Then $\mu^{(k)}=\mu^{(k-1)} \cup\left\{\left(i_{k}, \mu_{i_{k}}^{(k-1)}+1\right)\right\}$ (adding a box or dot in the $i_{k}$ th row of the Young diagram $\mu^{(k-1)}$ ), where $\mu^{(0)}=\emptyset$. In particular, $\mu^{(r)}=\mu$. Hence, the map sending $(S, T)$ to $(w(T) \rightarrow(\emptyset, S))$ gives a bijection [11, Corollary 4.11]

$$
\mathcal{B}_{v}^{\vee} \times \mathcal{B}_{\mu} \longrightarrow \mathcal{B}_{\mu, v}
$$

Next, for $(S, T) \in \mathcal{B}_{\mu} \times \mathcal{B}_{v}^{\vee}$, consider $((S, \emptyset) \leftarrow w(T))$. Suppose that $w(T)=$ $w_{1} \ldots w_{r}$ and $\left((S, \emptyset) \leftarrow w_{1} \ldots w_{k}\right) \in \mathcal{B}_{\mu^{(k)}, v^{(k)}}$ for $1 \leq k \leq r$. Let $\left(i_{k}, j_{k}\right) \in v$ correspond to $w_{k}$ in $T(1 \leq k \leq r)$. Define $U$ to be the tableau of shape $v$ such that for 
$1 \leq k \leq r$

$$
U\left(i_{k}, j_{k}\right)= \begin{cases}i, & \text { if } \mu^{(k)}=\mu^{(k-1)} \backslash\left\{\left(i, \mu_{i}^{(k-1)}\right)\right\}, \\ -i, & \text { if } v^{(k)}=v^{(k-1)} \cup\left\{\left(i, v_{i}^{(k-1)}+1\right)\right\},\end{cases}
$$

where $\mu^{(0)}=\mu$ and $v^{(0)}=\emptyset$. Then $U=U_{+} * U_{-}$, where $U_{+} \in S S T_{\mathbb{N}}(\lambda)$ and $U_{-} \in$ $S S T_{-\mathbb{N}}(\nu / \lambda)$ for some $\lambda \subset v$. Let $\sigma=\mu^{(r)}$ and $\tau=v^{(r)}$. We have $\imath\left(U_{+}\right) \in \mathbf{L} \mathbf{R}_{\sigma \lambda}^{\mu}$ and $U_{-} \in \overline{\mathbf{L R}}_{\lambda \tau}^{v}$, hence ${ }_{j}\left(U_{-}\right)_{R} \in \mathbf{L} \mathbf{R}_{\tau \lambda}^{v}$ (see Sects. 2.2 and 2.3). Therefore, we have a bijection [12, Proposition 4.3]

$$
\mathcal{B}_{\mu} \times \mathcal{B}_{v}^{\vee} \longrightarrow \bigsqcup_{\lambda, \sigma, \tau} \mathcal{B}_{\sigma, \tau} \times \mathbf{L R}_{\sigma \lambda}^{\mu} \times \mathbf{L R}_{\tau \lambda}^{\nu},
$$

where $(S, T)$ is mapped to $\left(((S, \emptyset) \leftarrow w(T)), l\left(U_{+}\right), J\left(U_{-}\right)_{R}\right)$. Now, we have

$$
\begin{aligned}
& \bigsqcup_{\lambda, \sigma, \tau} \mathcal{B}_{\sigma, \tau} \times \mathbf{L R}_{\sigma \lambda}^{\mu} \times \mathbf{L R}_{\tau \lambda}^{\nu} \\
& \stackrel{1-1}{\longleftrightarrow} \bigsqcup_{\lambda, \sigma, \tau} \mathcal{B}_{\tau}^{\vee} \times \mathcal{B}_{\sigma} \times \mathbf{L} \mathbf{R}_{\sigma \lambda}^{\mu} \times \mathbf{L} \mathbf{R}_{\tau \lambda}^{v} \quad \text { by (3.1) } \\
& \stackrel{1-1}{\longleftrightarrow} \bigsqcup_{\lambda, \sigma, \tau} \mathcal{B}_{\tau}^{\vee} \times \mathbf{L} \mathbf{R}_{\tau \lambda}^{v} \times \mathcal{B}_{\sigma} \times \mathbf{L} \mathbf{R}_{\sigma \lambda}^{\mu} \\
& \stackrel{1-1}{\longleftrightarrow} \bigsqcup_{\lambda \subset \mu, \nu} \mathcal{B}_{\nu / \lambda}^{\vee} \times \mathcal{B}_{\mu / \lambda}
\end{aligned}
$$

Hence, we obtain the following bijection [12, Proposition 5.1]:

$$
\mathcal{B}_{\mu} \times \mathcal{B}_{v}^{\vee} \longrightarrow \bigsqcup_{\lambda \subset \mu, v} \mathcal{B}_{v / \lambda}^{\vee} \times \mathcal{B}_{\mu / \lambda}
$$

\section{Plactic algebra}

4.1 A plactic algebra for $\mathfrak{g l}_{>0}$

Let $t$ be an indeterminate. Define $U(t)$ to be an associative $\mathbb{Q}\left[t, t^{-1}\right]$-algebra with unity generated by $u_{i}$ and $u_{i} \vee(i \in \mathbb{N})$ subject to the following relations:

$$
\begin{aligned}
& u_{i} u_{j} u_{k}=u_{i} u_{k} u_{j}, \quad u_{k^{\vee}} u_{j} \vee u_{i \vee}=u_{j} \vee u_{k^{\vee}} u_{i \vee} \quad(j \leq i<k), \\
& u_{i} u_{j} u_{k}=u_{j} u_{i} u_{k}, \quad u_{k^{\vee}} u_{j} \vee u_{i} \vee=u_{k^{\vee}} u_{i^{\vee}} u_{j^{\vee}} \quad(j<k \leq i) \text {, } \\
& u_{i+1} u_{(i+1)^{\vee}}=u_{i} \vee u_{i} \quad(i \geq 1), \quad u_{1} u_{1 \vee}=t, \\
& u_{i} u_{j \vee}=u_{j \vee} u_{i} \quad(i \neq j) .
\end{aligned}
$$

Let $U(t)_{+}$(resp. $U(t)_{-}$) be the subalgebra of $U(t)$ generated by $u_{i}$ (resp. $u_{i^{\vee}}$ ) for $i \in \mathbb{N}$. Then $\mathcal{U}(t)_{ \pm}$is isomorphic to the usual plactic algebra for $\mathfrak{g l}_{>0}$ over $\mathbb{Q}\left[t, t^{-1}\right]$ [14], where the first two relations in (4.1) are Knuth relations. 
Let $\mathcal{W}$ be the set of finite words with letters in $\mathbb{N} \cup \mathbb{N}^{\vee}$. For $w=w_{1} \cdots w_{r} \in \mathcal{W}$, put $u_{w}=u_{w_{1}} \cdots u_{w_{r}} \in \mathcal{U}(t)$, where we assume that $u_{\emptyset}=1$ for the empty word $\emptyset$. It is well known that if $w \in \mathcal{W}_{\mathbb{N}}\left(\right.$ resp. $\mathcal{W}_{\mathbb{N}^{\vee}}$ ), then there exists a unique $T \in \mathcal{B}_{\mu}$ (resp. $\left.\mathcal{B}_{\mu}^{\vee}\right)$ such that $u_{w}=u_{w(T)}$.

For a skew Young diagram $\lambda / \mu$ and $T \in \mathcal{B}_{\lambda / \mu}$ or $\mathcal{B}_{\lambda / \mu}^{\vee}$, we let $u_{T}=u_{w(T)}$, and for $\mu, v \in \mathcal{P}$ and $(S, T) \in \mathcal{B}_{\mu, v}$, we let $u_{(S, T)}=u_{S} u_{T}$.

Lemma 4.1 For $p, q \geq 1$, let $S \in \mathcal{B}_{\left(1^{p}\right)}$ and $T \in \mathcal{B}_{\left(1^{q}\right)}^{\vee}$ be given and let $\left(S^{\prime}, T^{\prime}\right)=$ $(w(S) \rightarrow(\emptyset, T)) \in \mathcal{B}_{\left(1^{p}\right),\left(1^{q}\right)}$. Then $u_{T} u_{S}=u_{\left(S^{\prime}, T^{\prime}\right)}$.

Proof It is straightforward to check it from (3.1) and (4.1).

Lemma 4.2 For $p, q \geq 1$, let $S \in \mathcal{B}_{\left(1^{p}\right)}$ and $T \in \mathcal{B}_{\left(1^{q}\right)}^{\vee}$ be given with $w(S)=$ $w_{1}^{+} \ldots w_{p}^{+}$and $w(T)=w_{q}^{-} \ldots w_{1}^{-}$. Suppose that there exists $k \geq 1$ such that $\left|\left\{i \mid w_{i}^{+} \leq k\right\}\right|+\left|\left\{j \mid w_{j}^{-} \geq k^{\vee}\right\}\right|>k$. If $w_{i}^{+}=k$ and $w_{j}^{-}=k^{\vee}$ for some $i$ and $j$, and $\left(S, T^{\prime}\right) \in \mathcal{B}_{\left(1^{p}\right),\left(1^{q-1}\right)}$, where $T^{\prime}$ is obtained from $T$ by removing $k^{\vee}$, then

$$
u_{S} u_{T}=t u_{w_{1}^{+} \ldots w_{i}^{+} \ldots w_{p}^{+}} u_{w_{q}^{-} \ldots \widehat{w_{j}^{-}} \ldots w_{1}^{-}} .
$$

Proof We use induction on $p+q$. If $p+q=2$, then $k=1$ and $u_{w_{1}^{+}} u_{w_{1}^{-}}=u_{1} u_{1} \vee=t$.

Suppose that $p+q>2$. First we assume that $i<p$ or $j<q$. Then

$$
\begin{aligned}
w_{i+1}^{+} \ldots w_{p}^{+} & =\left(k+a_{1}\right) \ldots\left(k+a_{p-i}\right), \\
w_{q}^{-} \ldots w_{j+1}^{-} & =\left(k+b_{q-j}\right)^{\vee} \ldots\left(k+b_{1}\right)^{\vee},
\end{aligned}
$$

for some $1 \leq a_{1}<\cdots<a_{p-i}$ and $1 \leq b_{1}<\cdots<b_{q-j}$. Also it follows from our hypothesis that $i+j=k+1$, and hence we can choose $(\bar{S}, \bar{T}) \in \mathcal{B}_{\left(1^{p-i}\right),\left(1^{q-j}\right)}$ such that $w(\bar{S})=a_{1} \ldots a_{p-i}$ and $w(\bar{T})=b_{q-j}^{\vee} \ldots b_{1}^{\vee}$. By Lemma 4.1, there exists $\left(\bar{T}^{\prime}, \bar{S}^{\prime}\right) \in \mathcal{B}_{\left(1^{q-j}\right)}^{\vee} \times \mathcal{B}_{\left(1^{p-i}\right)}$ with $w\left(\bar{S}^{\prime}\right)=a_{1}^{\prime} \ldots a_{p-i}^{\prime}$ and $w\left(\bar{T}^{\prime}\right)=\left(b_{q-j}^{\prime}\right)^{\vee} \ldots\left(b_{1}^{\prime}\right)^{\vee}$ such that $u_{\bar{T}^{\prime}} u_{\bar{S}^{\prime}}=u_{(\bar{S}, \bar{T})}=u_{\bar{S}} u_{\bar{T}}$. This implies that

$$
u_{w_{i+1}^{+} \ldots w_{p}^{+}} u_{w_{q}^{-} \ldots w_{j+1}^{-}}=u_{\left(k+b_{q-j}^{\prime}\right)^{\vee}} \ldots u_{\left(k+b_{1}^{\prime}\right)} \vee u_{\left(k+a_{1}^{\prime}\right)} \ldots u_{\left(k+a_{p-i}^{\prime}\right)} .
$$

Since $w_{i}^{+}=k<k+b_{1}^{\prime}$ and $w_{j}^{-}=k^{\vee}>\left(k+a_{1}^{\prime}\right)^{\vee}$, we have

$$
u_{S} u_{T}=u_{\left(k+b_{q-j}^{\prime}\right)^{\vee} \ldots\left(k+b_{1}^{\prime}\right)^{\vee}}\left(u_{w_{1}^{+}} \ldots u_{w_{i}^{+}} u_{w_{j}^{-}} \ldots u_{w_{1}^{-}}\right) u_{\left(k+a_{1}^{\prime}\right) \ldots\left(k+a_{p-i}^{\prime}\right)},
$$

and by induction hypothesis,

$$
\begin{aligned}
u_{S} u_{T} & =t u_{\left(k+b_{q-j}^{\prime}\right)^{\vee} \ldots\left(k+b_{1}^{\prime}\right)^{\vee}}\left(u_{w_{1}^{+}} \ldots \widehat{u_{w_{i}^{+}}} \widehat{u_{w_{j}^{-}}} \ldots u_{w_{1}^{-}}\right) u_{\left(k+a_{1}^{\prime}\right) \ldots\left(k+a_{p-i}^{\prime}\right)} \\
& =t u_{w_{1}^{+}} \ldots \widehat{u_{w_{i}^{+}}} \ldots u_{w_{p}^{+}} u_{w_{q}^{-}} \ldots \widehat{u_{w_{j}^{-}}} \ldots u_{w_{1}^{-}} .
\end{aligned}
$$


Next, we assume that $i=p$ and $j=q$, that is, $w_{p}^{+}=k$ and $w_{q}^{-}=k^{\vee}$. Note that $p+q=k+1$. If $w_{p-1}^{+} \neq k-1$ and $w_{q-1}^{-} \neq(k-1)^{\vee}$, then

$$
\left|\left\{i \mid w_{i}^{+} \leq k-2\right\}\right|+\left|\left\{j \mid w_{j}^{-} \geq(k-2)^{\vee}\right\}\right|=p+q-2=k-1>k-2,
$$

which contradicts the fact that $\left(S, T^{\prime}\right) \in \mathcal{B}_{\left(1^{p}\right),\left(1^{q-1}\right)}$. So we also assume that either $w_{p-1}^{+}=k-1$ or $w_{q-1}^{-}=(k-1)^{\vee}$.

Case 1. Suppose that $w_{p-1}^{+} \neq k-1$ and $w_{q-1}^{-}=(k-1)^{\vee}$. We have

$$
\begin{aligned}
u_{S} u_{T} & =u_{w_{1}^{+}} \ldots u_{w_{p-1}^{+}} u_{k} u_{k^{\vee}} u_{w_{q-1}^{-}} \ldots u_{w_{1}^{-}} \\
& =u_{w_{1}^{+}} \ldots u_{w_{p-1}^{+}} u_{(k-1)^{\vee}} u_{k-1} u_{w_{q-1}^{-}} \ldots u_{w_{1}^{-}} \\
& =u_{(k-1)} \vee u_{w_{1}^{+}} \ldots u_{w_{p-1}^{+}} u_{k-1} u_{(k-1)^{\vee}} u_{w_{q-2}^{-}} \ldots u_{w_{1}^{-}} \\
& =t u_{(k-1)^{\vee}} u_{w_{1}^{+}} \ldots u_{w_{p-1}^{+}} u_{w_{q-2}^{-}} \ldots u_{w_{1}^{-}} \\
& =t u_{w_{1}^{+}} \ldots u_{w_{p-1}^{+}} u_{(k-1)} \vee u_{w_{q-2}^{-}} \ldots u_{w_{1}^{-}} \\
& =t u_{w_{1}^{+}} \ldots u_{w_{p-1}^{+}} u_{w_{q-1}^{-}} \ldots u_{w_{1}^{-}}
\end{aligned}
$$

where we use induction hypothesis in the third line.

Case 2. Suppose that $w_{p-1}^{+}=k-1$ and $w_{q-1}^{-} \neq(k-1)^{\vee}$. By almost the same argument as in Case 1, we have

$$
u_{S} u_{T}=t u_{w_{1}^{+}} \ldots u_{w_{p-1}^{+}} u_{w_{q-1}^{-}} \ldots u_{w_{1}^{-}} .
$$

Case 3. Suppose that $w_{p-1}^{+}=k-1$ and $w_{q-1}^{-}=(k-1)^{\vee}$. We have

$$
\begin{aligned}
u_{S} u_{T} & =u_{w_{1}^{+}} \ldots u_{w_{p-1}^{+}} u_{k} u_{k} \vee u_{w_{q-1}^{-}} \ldots u_{w_{1}^{-}} \\
& =u_{w_{1}^{+}} \ldots u_{w_{p-1}^{+}} u_{(k-1)} \vee u_{k-1} u_{w_{q-1}^{-}} \ldots u_{w_{1}^{-}} \\
& =u_{(k-a)^{\vee}} u_{v_{1}} \ldots u_{v_{p-2}} u_{k-2} u_{k-1} u_{w_{q-1}^{-}} \ldots u_{w_{1}^{-}},
\end{aligned}
$$

for some $1 \leq a<k$ and $1 \leq v_{1}<\cdots<v_{p-2}<k-2$. So by induction hypothesis,

$$
\begin{aligned}
u_{S} u_{T} & =u_{(k-a)^{\vee}} u_{v_{1}} \ldots u_{v_{p-2}} u_{k-2} u_{k-1} u_{(k-1)^{\vee}} u_{w_{q-2}^{-}} \ldots u_{w_{1}^{-}} \\
& =t u_{(k-a)^{\vee}} u_{v_{1}} \ldots u_{v_{p-2}} u_{k-2} u_{w_{q-2}^{-}} \ldots u_{w_{1}^{-}} \\
& =t u_{w_{1}^{+}} \ldots u_{w_{p-2}^{+}} u_{k-1} u_{(k-1)^{\vee}} u_{w_{q-2}^{-}} \ldots u_{w_{1}^{-}} \\
& =t u_{w_{1}^{+}} \ldots u_{w_{p-1}^{+}} u_{w_{q-1}^{-}} \ldots u_{w_{1}^{-}}
\end{aligned}
$$

This completes the induction. 
Lemma 4.3 Let $\mu, v \in \mathcal{P}$ be given. For $a \in \mathbb{N}$ and $(S, T) \in \mathcal{B}_{\mu, \nu}$,

(1) $u_{(S, T)} u_{a}=u_{(a \rightarrow(S, T))}$,

(2) $u_{(S, T)} u_{a^{\vee}}=t^{\epsilon} u_{\left((S, T) \leftarrow a^{\vee}\right)}$, where $\epsilon=0,1$.

Proof We keep the notations in Sect. 3.1. Consider $(a \rightarrow(S, T))=\left(S^{\prime}, T^{\prime}\right)$. Let $\left(T^{\prime}, a^{\prime}\right)$ be the pair obtained by the first step in the definition of $a \rightarrow(S, T)$. It is straightforward to check that $u_{T} u_{a}=u_{a^{\prime}} u_{T^{\prime}}$. Since $S^{\prime}=\left(a^{\prime} \rightarrow S\right)$, which is a usual column insertion, we have $u_{S^{\prime}}=u_{S} u_{a^{\prime}}$. Hence

$$
u_{(S, T)} u_{a}=u_{S} u_{T} u_{a}=u_{S} u_{a^{\prime}} u_{T^{\prime}}=u_{S^{\prime}} u_{T^{\prime}}=u_{(a \rightarrow(S, T))} .
$$

Next, consider $\left((S, T) \leftarrow a^{\vee}\right)=\left(S^{\prime}, T^{\prime}\right)$. If the pair $\left(S,\left(T^{\vee} \leftarrow a\right)^{\vee}\right)$ satisfies the condition (2) in Definition 3.1, then $\left(S^{\prime}, T^{\prime}\right)=\left(S,\left(T^{\vee} \leftarrow a\right)^{\vee}\right)$, which implies that $u_{S}=u_{S^{\prime}}$ and $u_{T} u_{a^{\vee}}=u_{T^{\prime}}$. Hence, $u_{(S, T)} u_{a^{\vee}}=u_{\left((S, T) \leftarrow a^{\vee}\right)}$.

Suppose that there exists $j$ such that $a_{j}=k$ is bumped out of the $(j-1)$-st row in the row insertion of $a$ into $T^{\vee}$ and the insertion of $a_{j}$ into the $j$ th row violates the condition (2) in Definition 3.1.

Let $T^{\prime \prime}=\left(T^{\vee} \leftarrow a\right)^{\vee}$. Suppose that $w(S)=\widetilde{w}^{+} w^{+}$and $w\left(T^{\prime \prime}\right)=w^{-} \widetilde{w}^{-}$, where $w^{+}=w_{1}^{+} \ldots w_{p}^{+}$is the subword corresponding to the leftmost column of $S$ and $w^{-}=$ $w_{q}^{-} \ldots w_{1}^{-}$is the subword corresponding to the rightmost column of $T^{\prime \prime}$ reading from top to bottom. Note that $w_{j}^{-}=k^{\vee}$. Suppose that $w_{i}^{+}=k$. By Lemma 4.2, we have

$$
u_{w^{+}} u_{w^{-}}=t u_{w_{1}^{+} \ldots \widehat{w_{i}^{+}} \ldots w_{p}^{+}} u_{w_{q}^{-} \ldots \widehat{w_{j}^{-}} \ldots w_{1}^{-}} .
$$

Note that $w\left(T^{\prime}\right)=w_{q}^{-} \ldots \widehat{w_{j}^{-}} \ldots w_{1}^{-} \widetilde{w}^{-}$. Recalling that $S^{\prime}$ is obtained by playing the jeu de taquin after removing $k$ in the first column of $S$, it follows that $u_{S^{\prime}}=$ $u_{\widetilde{w}^{+}} u_{w_{1}^{+} \ldots \widehat{w_{i}^{+}} \ldots w_{p}^{+}}$. Therefore,

$$
\begin{aligned}
u_{(S, T)} u_{a^{\vee}} & =u_{S} u_{T} u_{a^{\vee}}=u_{S} u_{T^{\prime \prime}} \\
& =u_{\widetilde{w}^{+}} u_{w^{+}} u_{w^{-}} u_{\widetilde{w}^{-}} \\
& =t u_{\widetilde{w}^{+}} u_{w_{1}^{+} \ldots \widehat{w_{i}^{+}} \ldots w_{p}^{+}} u_{w_{q}^{-} \ldots \widehat{w_{j}^{-}} \ldots w_{1}^{-}} u_{\widetilde{w}^{-}} \\
& =t u_{S^{\prime}} u_{T^{\prime}}=t u_{\left((S, T) \leftarrow a^{\vee}\right) .} .
\end{aligned}
$$

Now, we obtain the following immediately.

Proposition 4.4 For $w=w_{1} \ldots w_{r} \in \mathcal{W}$, there exists $(S, T) \in \mathcal{B}_{\mu, v}$ such that $u_{w}=$ $t^{\epsilon} u_{(S, T)}$ where $\epsilon=r-|\mu|-|\nu|$.

Corollary 4.5 The set $\left\{u_{(S, T)} \mid(S, T) \in \mathcal{B}_{\mu, v}, \mu, v \in \mathcal{P}\right\}$ spans $U(t)$ over $\mathbb{Q}\left[t, t^{-1}\right]$.

The uniqueness of $(S, T)$ in Proposition 4.4 and the linear independence of the spanning set in Corollary 4.5 will be proved in Sect. 4.2. 


\subsection{Crystal equivalence}

Let $P=P_{\mathbb{N}}=\bigoplus_{i \in \mathbb{N}} \mathbb{Z} \epsilon_{i}$ be the weight lattice of $\mathfrak{g l}_{>0}$ with a symmetric bilinear form $(\cdot, \cdot)$ given by $\left(\epsilon_{i}, \epsilon_{j}\right)=\delta_{i j}$. Let $\left\{\alpha_{i}=\epsilon_{i}-\epsilon_{i+1} \mid i \in \mathbb{N}\right\}$ be the set of simple roots of $\mathfrak{g l}_{>0}$. A (normal) $\mathfrak{g l}_{>0}$-crystal is a set $B$ together with the maps wt: $B \rightarrow P$, $\varepsilon_{i}, \varphi_{i}: B \rightarrow \mathbb{Z}_{\geq 0}$ and $\widetilde{e}_{i}, \widetilde{f}_{i}: B \rightarrow B \cup\{\mathbf{0}\}(i \in \mathbb{N})$ such that for $b, b^{\prime} \in B$

(1) $\varphi_{i}(b)=\left(\operatorname{wt}(b), \alpha_{i}\right)+\varepsilon_{i}(b)$,

(2) $\varepsilon_{i}(b)=\max \left\{k \mid \widetilde{e}_{i}^{k} b \neq \mathbf{0}\right\}$ and $\varphi_{i}(b)=\max \left\{k \mid \widetilde{f}_{i}^{k} b \neq \mathbf{0}\right\}$,

(3) $\operatorname{wt}\left(\widetilde{e}_{i} b\right)=\operatorname{wt}(b)+\alpha_{i}$ if $\widetilde{e}_{i} b \neq \mathbf{0}$, and $\operatorname{wt}\left(\widetilde{f}_{i} b\right)=\operatorname{wt}(b)-\alpha_{i}$ if $\tilde{f}_{i} b \neq \mathbf{0}$,

(4) $\widetilde{f}_{i} b=b^{\prime}$ if and only if $b=\widetilde{e}_{i} b^{\prime}$,

where $\mathbf{0}$ is a formal symbol (cf. [7]). Note that $B$ is equipped with a colored oriented graph structure, where $b \stackrel{i}{\rightarrow} b^{\prime}$ if and only if $b^{\prime}=\widetilde{f}_{i} b$ for $b, b^{\prime} \in B$ and $i \in \mathbb{N}$. The dual crystal $B^{\vee}$ of $B$ is defined to be the set $\left\{b^{\vee} \mid b \in B\right\}$ with $\operatorname{wt}\left(b^{\vee}\right)=-\mathrm{wt}(b)$, $\widetilde{e}_{i}\left(b^{\vee}\right)=\left(\widetilde{f_{i}} b\right)^{\vee}$ and $\widetilde{f_{i}}\left(b^{\vee}\right)=\left(\widetilde{e}_{i} b\right)^{\vee}$ for $b \in B$ and $i \in \mathbb{N}$. We assume that $\mathbf{0}^{\vee}=\mathbf{0}$. Note that $\mathbb{N}$ is naturally equipped with a $\mathfrak{g l}_{>0}$-crystal structure;

$$
1 \stackrel{1}{\longrightarrow} 2 \stackrel{2}{\longrightarrow} 3 \stackrel{3}{\longrightarrow} \cdots
$$

with $\operatorname{wt}(i)=\epsilon_{i}(i \in \mathbb{N})$, while $\mathbb{N}^{\vee}$ is its dual.

For $\mathfrak{g l}_{>0}$-crystals $B_{1}$ and $B_{2}$, a tensor product $B_{1} \otimes B_{2}$ is defined to be $B_{1} \times B_{2}$ as a set with elements denoted by $b_{1} \otimes b_{2}$, where

$$
\begin{gathered}
\operatorname{wt}\left(b_{1} \otimes b_{2}\right)=\operatorname{wt}\left(b_{1}\right)+\operatorname{wt}\left(b_{2}\right), \\
\widetilde{e}_{i}\left(b_{1} \otimes b_{2}\right)= \begin{cases}\widetilde{e}_{i} b_{1} \otimes b_{2}, & \text { if } \varphi_{i}\left(b_{1}\right) \geq \varepsilon_{i}\left(b_{2}\right), \\
b_{1} \otimes \widetilde{e}_{i} b_{2}, & \text { if } \varphi_{i}\left(b_{1}\right)<\varepsilon_{i}\left(b_{2}\right),\end{cases} \\
\tilde{f}_{i}\left(b_{1} \otimes b_{2}\right)= \begin{cases}\tilde{f}_{i} b_{1} \otimes b_{2}, & \text { if } \varphi_{i}\left(b_{1}\right)>\varepsilon_{i}\left(b_{2}\right), \\
b_{1} \otimes \widetilde{f}_{i} b_{2}, & \text { if } \varphi_{i}\left(b_{1}\right) \leq \varepsilon_{i}\left(b_{2}\right),\end{cases}
\end{gathered}
$$

for $i \in \mathbb{N}$ and $b_{1} \otimes b_{2} \in B_{1} \otimes B_{2}$. Here we assume that $\mathbf{0} \otimes b_{2}=b_{1} \otimes \mathbf{0}=\mathbf{0}$. For example, $\mathcal{W}$ is a $\mathfrak{g l}_{>0}$-crystal, where each word $w_{1} \ldots w_{r}$ is identified with $w_{1} \otimes$ $\cdots \otimes w_{r}$ in a mixed $r$-tensor product of $\mathbb{N}$ and $\mathbb{N}^{\vee}$.

For $b_{i} \in B_{i}(i=1,2)$, we say that $b_{1}$ is equivalent to $b_{2}$, and write $b_{1} \equiv b_{2}$ if $\operatorname{wt}\left(b_{1}\right)=\mathrm{wt}\left(b_{2}\right)$ and they generate the same $\mathbb{N}$-colored graph with respect to $\widetilde{e}_{i}, \widetilde{f}_{i}$ $(i \in \mathbb{N})$. We usually call $\equiv$ the crystal equivalence.

For a skew Young diagram $\lambda / \mu, \mathcal{B}_{\lambda / \mu}$ has a well-defined $\mathfrak{g l}_{>0}$-crystal structure such that $\tilde{x}_{i}(S)=S^{\prime}$ if $\tilde{x}_{i} w(S) \neq \mathbf{0}(i \in \mathbb{N}, x=e, f)$, where $S^{\prime}$ is the unique tableau in $\mathcal{B}_{\lambda / \mu}$ with $w\left(S^{\prime}\right)=\tilde{x}_{i} w(S)$, and $\tilde{x}_{i}(S)=\mathbf{0}$ otherwise [9]. We regard $\mathcal{B}_{\lambda / \mu}^{\vee}$ as the dual of $\mathcal{B}_{\lambda / \mu}$. Moreover, for $\mu, v \in \mathcal{P}, \mathcal{B}_{\mu, v} \cup\{\boldsymbol{0}\} \subset\left(\mathcal{B}_{\mu} \otimes \mathcal{B}_{v}^{\vee}\right) \cup\{\boldsymbol{0}\}$ is invariant under $\widetilde{e}_{i}, f_{i}(i \in \mathbb{N})$, and hence a $\mathfrak{g l}_{>0}$-crystal, which is connected as a graph [11, Proposition 3.4]. It is shown in [11, Theorem 3.5] that $\mathcal{B}_{\mu, v}$ is an extremal weight crystal which was introduced by Kashiwara [8]. 
Let $\mathcal{W}$ be an associative $\mathbb{Q}$-algebra with unity generated by the symbol $[w](w \in$ $\mathcal{W})$ subject to the relations;

$$
\begin{aligned}
{[w]\left[w^{\prime}\right] } & =\left[w w^{\prime}\right], \\
{[w] } & =\left[w^{\prime}\right], \quad \text { if } w \equiv w^{\prime},
\end{aligned}
$$

for $w, w^{\prime} \in \mathcal{W}$. Note that $[\emptyset]=1$ is the unity in $\mathcal{W}$, where $\emptyset$ is the empty word.

Lemma 4.6 The set

$$
\mathscr{B}=\left\{[w(S) w(T)] \mid(S, T) \in \mathcal{B}_{\mu, \nu}, \mu, v \in \mathcal{P}\right\}
$$

is a $\mathbb{Q}$-basis of $\mathcal{W}$.

Proof For $a \in \mathbb{N}$ and $(S, T) \in \mathcal{B}_{\mu, v}$, it is shown in [11, Lemma 4.4] that

$$
(a \rightarrow(S, T)) \equiv(S, T) \otimes a, \quad\left((S, T) \leftarrow a^{\vee}\right) \equiv(S, T) \otimes a^{\vee} .
$$

This implies that for $w \in \mathcal{W},[w]=[w(S) w(T)]$ for some $(S, T) \in \mathcal{B}_{\mu, \nu}$, and hence $\mathcal{W}$ is spanned by $\mathcal{B}$.

Now, suppose that

$$
\sum_{i=1}^{n} c_{i}\left[w\left(S^{(i)}\right) w\left(T^{(i)}\right)\right]=0
$$

for some $c_{i} \in \mathbb{Q}$ and $\left(S^{(i)}, T^{(i)}\right) \in \mathcal{B}_{\mu^{(i)}, v^{(i)}}(1 \leq i \leq n)$. Since $(S, T) \equiv\left(S^{\prime}, T^{\prime}\right)$ implies $(S, T)=\left(S^{\prime}, T^{\prime}\right)$ for $(S, T) \in \mathcal{B}_{\mu, \nu}$ and $\left(S^{\prime}, T^{\prime}\right) \in \mathcal{B}_{\sigma, \tau}$ [11, Lemma 5.1], we assume that $\left(S^{(i)}, T^{(i)}\right)$ 's are mutually different.

We use induction on $n$ to show that $c_{i}=0$ for $1 \leq i \leq n$. It is clear when $n=1$. Suppose that $n \geq 2$.

We claim that there exist $j_{1}, \ldots, j_{r}$ such that $\tilde{x}_{j_{1}} \ldots \tilde{x}_{j_{r}}\left(S^{(1)}, T^{(1)}\right)=\mathbf{0}$ but $\tilde{x}_{j_{1}} \cdots \tilde{x}_{j_{r}}\left(S^{(i)}, T^{(i)}\right) \neq \mathbf{0}$ for some $2 \leq i \leq n$, where $x$ denotes $e$ or $f$ for each $j_{k}$.

Consider $\left(S^{(i)}, T^{(i)}\right)(i=1,2)$ and suppose that $\tilde{x}_{j_{1}} \cdots \tilde{x}_{j_{r}}\left(S^{(1)}, T^{(1)}\right) \neq \mathbf{0}$ if and only if $\tilde{x}_{j_{1}} \cdots \tilde{x}_{j_{r}}\left(S^{(2)}, T^{(2)}\right) \neq \mathbf{0}$ for all $j_{1}, \ldots, j_{r}$. Then by applying suitable $\tilde{e}_{k}$ 's, we may assume that $S^{(i)}=H_{\mu^{(i)}}$ and $\left(T^{(i)}\right)^{\vee}(k, l) \geq p$ for $(k, l) \in v^{(i)}$, where $p \gg \ell\left(\mu^{(i)}\right)(i=1,2)$. Now, $\tilde{x}_{j_{1}} \cdots \tilde{x}_{j_{r}} H_{\mu^{(1)}} \neq \mathbf{0}$ if and only if $\tilde{x}_{j_{1}} \cdots \tilde{x}_{j_{r}} H_{\mu^{(2)}} \neq \mathbf{0}$ for all $1 \leq j_{1}, \ldots, j_{r} \leq p-2$ since $\tilde{x}_{j_{1}} \cdots \tilde{x}_{j_{r}} T^{(i)}=\mathbf{0}(i=1,2)$. This implies that $H_{\mu^{(1)}}=H_{\mu^{(2)}}$. Also, we regard $T^{(i)}(i=1,2)$ as elements in $\mathfrak{g l}_{>0}$-crystals (whose weight lattice is $\left.\bigoplus_{i \geq p} \mathbb{Z} \epsilon_{i}\right)$ with respect to $\widetilde{e}_{k}$ and $\widetilde{f}_{k}(k \geq p)$. Then $\tilde{x}_{j_{1}} \cdots \tilde{x}_{j_{r}} T^{(1)} \neq$ $\mathbf{0}$ if and only if $\tilde{x}_{j_{1}} \cdots \tilde{x}_{j_{r}} T^{(2)} \neq \mathbf{0}$ for all $j_{1}, \ldots, j_{r} \geq p$ since $\tilde{x}_{j_{1}} \cdots \tilde{x}_{j_{r}} H_{\mu^{(i)}}=\mathbf{0}$ $(i=1,2)$. This implies that $T^{(1)}=T^{(2)}$. Therefore, $\left(S^{(1)}, T^{(1)}\right)=\left(S^{(2)}, T^{(2)}\right)$, which is a contradiction. This proves our claim.

Note that $\tilde{x}_{i}(x=e, f, i \in \mathbb{N})$ acts on $\mathcal{W}$ by $\tilde{x}_{i}[w]=\left[\tilde{x}_{i} w\right]$, where we assume that $[\mathbf{0}]=0$. Hence by applying $X=\tilde{x}_{j_{1}} \cdots \tilde{x}_{j_{r}}$ to (4.3), we get

$$
\sum_{i=2}^{n} c_{i}\left[X w\left(S^{(i)}\right) w\left(T^{(i)}\right)\right]=\sum_{i=2}^{n} c_{i}\left[w\left(\bar{S}^{(i)}\right) w\left(\bar{T}^{(i)}\right)\right]=0
$$


for some $\left[w\left(\bar{S}^{(i)}\right) w\left(\bar{T}^{(i)}\right)\right] \in \mathscr{B}$. Here, we assume that $c_{i}=0$ if $X\left(w\left(S^{(i)}\right) w\left(T^{(i)}\right)\right)$ $=\mathbf{0}$. By induction hypothesis, we have $c_{2}=\cdots=c_{n}=0$, and hence $c_{1}=0$. Therefore, $\mathcal{B}$ is a $\mathbb{Q}$-basis of $\mathcal{W}$.

Theorem 4.7 Let $\mathcal{U}(1)$ be the $\mathbb{Q}$-algebra obtained from $\mathcal{U}(t)$ by specializing $t=1$. Then the assignment $u_{a} \mapsto[a]$ for $a \in \mathbb{N} \cup \mathbb{N}^{\vee}$ gives a $\mathbb{Q}$-algebra isomorphism

$$
U(1) \simeq \mathcal{W}
$$

Proof By (4.2), the relations in (4.1) when $t=1$ are preserved in $\mathcal{W}$ under the correspondence $u_{a} \mapsto[a]$. Hence there exists a $\mathbb{Q}$-algebra homomorphism $\psi: \mathcal{U}(1) \rightarrow \mathcal{W}$ sending $u_{a}$ to $[a]$ for $a \in \mathbb{N} \cup \mathbb{N}^{\vee}$. Since $\left\{u_{(S, T)} \mid(S, T) \in \mathcal{B}_{\mu, v}, \mu, v \in \mathcal{P}\right\}$ spans $\mathcal{U}(1)$ and $\psi\left(u_{(S, T)}\right)=[w(S) w(T)]$, it follows from Lemma 4.6 that $\psi$ is an isomorphism.

Corollary 4.8 The set

$$
\left\{u_{(S, T)} \mid(S, T) \in \mathcal{B}_{\mu, \nu}, \mu, v \in \mathcal{P}\right\}
$$

is a $\mathbb{Q}\left[t, t^{-1}\right]$-basis of $U(t)$.

Proof Note that $\left\{u_{(S, T)} \mid(S, T) \in \mathcal{B}_{\mu, \nu}, \mu, v \in \mathcal{P}\right\} \subset \mathcal{U}(1)$ is a $\mathbb{Q}$-basis of $\mathcal{U}(1)$ since it is mapped to $\mathscr{B}$ by Theorem 4.7. Then it is not difficult to check that $\left\{u_{(S, T)} \mid(S, T) \in \mathcal{B}_{\mu, v}, \mu, v \in \mathcal{P}\right\} \subset \mathcal{U}(t)$ is linearly independent over $\mathbb{Q}\left[t, t^{-1}\right]$ and hence a $\mathbb{Q}\left[t, t^{-1}\right]$-basis of $U(t)$ since $U(t)$ is a $\mathbb{Q}\left[t, t^{-1}\right]$-submodule of a $\mathbb{Q}(t)$-vector space $\mathbb{Q}(t) \otimes_{\mathbb{Q}\left[t, t^{-1}\right]} \mathcal{U}(t)$.

Corollary 4.9 For $w \in \mathcal{W}$, there exist unique $(S, T) \in \mathcal{B}_{\mu, v}$ and $\epsilon \in \mathbb{Z}_{\geq 0}$ such that $u_{w}=t^{\epsilon} u_{(S, T)}$.

\subsection{Non-commutative Schur functions}

Let $\widehat{U(t)}=\bigoplus_{n \geq 0} \widehat{U(t)}_{n}$, where $\widehat{U(t)}_{n}$ is the completion of $\mathbb{Q}\left[t, t^{-1}\right]$-submodule of $\mathcal{U}(t)$ spanned by $\left\{(S, T)\left|(S, T) \in \mathcal{B}_{\mu, \nu},\right| \mu|+| \nu \mid=n\right\}$. For a skew Young diagram $\lambda / \mu$, let

$$
s_{\lambda / \mu}(\mathbf{u})=\sum_{S \in \mathcal{B}_{\lambda / \mu}} u_{S}, \quad s_{\lambda / \mu}^{\vee}(\mathbf{u})=\sum_{S \in \mathcal{B}_{\lambda / \mu}^{\vee}} u_{S} \in \widehat{\mathcal{U}(t)},
$$

which are plactic skew Schur functions in $u_{i}$ 's and $u_{i} \vee$ 's, respectively.

Let $\Lambda(t)$ be the algebra of symmetric functions in $\mathbf{x}=\mathbf{x}_{\mathbb{N}}$ over $\mathbb{Q}\left[t, t^{-1}\right]$. Then $\left\{s_{(k)}(\mathbf{u}) \mid k \geq 0\right\}$ (resp. $\left.\left\{s_{(k)}^{\vee}(\mathbf{u}) \mid k \geq 0\right\}\right)$ generates the subalgebra $\delta(t)_{ \pm}$of $\widehat{\mathcal{U}(t)}$ isomorphic to $\Lambda(t)$ [14], where $s_{(k)}(\mathbf{u})$ (resp. $s_{(k)}^{\vee}(\mathbf{u})$ ) corresponds to the $k$ th complete symmetric function $h_{k}(\mathbf{x})=s_{(k)}(\mathbf{x})$, and $\left\{s_{\lambda}(\mathbf{u}) \mid \lambda \in \mathcal{P}\right\}\left(\operatorname{resp} .\left\{s_{\lambda}^{\vee}(\mathbf{u}) \mid \lambda \in \mathcal{P}\right\}\right)$ is a $\mathbb{Q}\left[t, t^{-1}\right]$-basis of $\delta(t)_{+}\left(\operatorname{resp} . \delta(t)_{-}\right)$. 
We define

$$
s_{\mu, v}(\mathbf{u})=\sum_{(S, T) \in \mathcal{B}_{\mu, v}} u_{(S, T)}
$$

for $\mu, v \in \mathcal{P}$ and let

$$
s(t)=\sum_{\mu, \nu \in \mathcal{P}} \mathbb{Q}\left[t, t^{-1}\right] s_{\mu, v}(\mathbf{u}) \subset \widehat{U(t)} .
$$

Lemma 4.10 For $\mu, v \in \mathcal{P}$, we have

$$
s_{\mu}(\mathbf{u}) s_{v}^{\vee}(\mathbf{u})=\sum_{\lambda \subset \mu, v} t^{|\lambda|} s_{v / \lambda}^{\vee}(\mathbf{u}) s_{\mu / \lambda}(\mathbf{u})=\sum_{\lambda, \sigma, \tau} t^{|\lambda|} c_{\lambda \sigma}^{\mu} c_{\lambda \tau}^{\nu} s_{\sigma, \tau}(\mathbf{u}) .
$$

Proof By (3.1) and Lemma 4.3(1), we have $s_{\mu, v}(\mathbf{u})=s_{v}^{\vee}(\mathbf{u}) s_{\mu}(\mathbf{u})$. The identity follows from (3.3) and Lemma 4.3(2).

Proposition 4.11 $\&(t)$ is a $\mathbb{Q}\left[t, t^{-1}\right]$-algebra with a basis $\left\{s_{\mu, v}(\mathbf{u}) \mid \mu, v \in \mathcal{P}\right\}$, where

$$
s_{\mu, \nu}(\mathbf{u}) s_{\sigma, \tau}(\mathbf{u})=\sum_{\zeta, \eta}\left(\sum_{\alpha, \beta, \gamma} t^{|\beta|} c_{\sigma \alpha}^{\zeta} c_{\alpha \beta}^{\mu} c_{\beta \gamma}^{\tau} c_{\gamma \nu}^{\eta}\right) s_{\zeta, \eta}(\mathbf{u})
$$

for $\mu, v, \sigma, \tau \in \mathcal{P}$.

Proof In fact, $\left\{s_{\mu, \nu}(\mathbf{u}) \mid \mu, v \in \mathscr{P}\right\}$ is linearly independent by Lemma 4.8, and hence a basis of $\delta(t)$. Combining Lemma 4.10 with the usual Littlewood-Richardson rule (2.1) for $s_{\mu}$ (u)'s and $s_{v}^{\vee}(\mathbf{u})$ 's, we obtain the above identity. Since the sum on the right hand side is finite, $\delta(t)$ has a well-defined multiplication and hence is a $\mathbb{Q}\left[t, t^{-1}\right]$ algebra.

\subsection{Heisenberg algebra}

Let $\mathscr{H}(t)$ be an associative $\mathbb{Q}\left[t, t^{-1}\right]$-algebra with unity generated by $B_{n}(n \in \mathbb{Z} \backslash\{0\})$ subject to the relations

$$
B_{k} B_{l}-B_{l} B_{k}=k t^{k} \delta_{k+l, 0} .
$$

For $k \geq 1$, let $p_{k}(\mathbf{u}) \in \delta(t)_{+}\left(\right.$resp. $\left.p_{k}^{\vee}(\mathbf{u}) \in \delta(t)_{-}\right)$correspond to the $k$ th power sum symmetric function $p_{k}(\mathbf{x}) \in \Lambda(t)$.

Proposition 4.12 The assignment $p_{k}(\mathbf{u}) \mapsto B_{k} p_{k}^{\vee}(\mathbf{u}) \mapsto B_{-k}$ for $k \geq 1$ gives a $\mathbb{Q}\left[t, t^{-1}\right]$-algebra isomorphism

$$
\delta(t) \simeq \mathscr{H}(t) .
$$


Proof Put $h_{k}(\mathbf{u})=s_{(k)}(\mathbf{u})$ and $h_{k}^{\vee}(\mathbf{u})=s_{(k)}^{\vee}(\mathbf{u})$ for $k \geq 0$ (note that $h_{0}(\mathbf{u})=h_{0}^{\vee}(\mathbf{u})$ $=1)$. By Lemma 4.10, we have

$$
h_{s}(\mathbf{u}) h_{r}^{\vee}(\mathbf{u})=\sum_{i=0}^{m} t^{i} h_{r-i}^{\vee}(\mathbf{u}) h_{s-i}(\mathbf{u})
$$

for $r, s \geq 0$, where $m=\min \{r, s\}$. We may view $\&(t)$ as an algebra generated by $\left\{h_{k}^{\vee}(\mathbf{u}), h_{k}(\mathbf{u}) \mid k \geq 0\right\}$ with the defining relations (4.4). Since

$$
h_{r}(\mathbf{u})=\sum_{|\lambda|=r} \frac{1}{z_{\lambda}} p_{\lambda}(\mathbf{u}),
$$

where $z_{\lambda}=\prod_{i \geq 1} i^{m_{i}(\lambda)} m_{i}(\lambda)$ ! and $m_{i}(\lambda)=\left|\left\{k \mid \lambda_{k}=i\right\}\right|$, we obtain

$$
p_{k}(\mathbf{u}) p_{l}^{\vee}(\mathbf{u})-p_{l}^{\vee}(\mathbf{u}) p_{k}(\mathbf{u})=k t^{k} \delta_{k, l}
$$

for $k, l \geq 1$ by using the same argument as in [13, Corollary 8]. This implies that there exists an isomorphism $\psi: \mathscr{H}(t) \rightarrow \&(t)$ sending $B_{-k}\left(\right.$ resp. $\left.B_{k}\right)$ to $p_{k}^{\vee}(\mathbf{u})$ (resp. $\left.p_{k}(\mathbf{u})\right)$ for $k \geq 1$.

Remark 4.13 Regarding $\&(0)$ and $\delta(1)$ as $\mathbb{Q}$-algebras generated by $h_{k}(\mathbf{u})$ and $h_{k}^{\vee}(\mathbf{u})$ $(k \geq 0)$, we have $\delta(0) \simeq \Lambda \otimes \Lambda$, and $\&(1) \simeq\left\langle\frac{\partial}{\partial p_{k}}, p_{k} \mid k \geq 1\right\rangle \subset \operatorname{End}_{\mathbb{Q}}(\Lambda)$, where $\Lambda$ is the algebra of symmetric functions in $\mathbf{x}$ over $\mathbb{Q}$ and $p_{k}$ is the operator on $\Lambda$ induced from the multiplication by $p_{k}(\mathbf{x})$. Therefore, we may view $\delta(t)$ as an algebra interpolating the algebra of double symmetric functions and the Weyl algebra of infinite rank.

\section{Knuth correspondence and Cauchy identity}

\subsection{Main result}

Let $\mathbb{A}$ and $\mathbb{B}$ be linearly ordered $\mathbb{Z}_{2}$-graded sets. For $A \in \mathcal{M}_{\mathbb{A}, \mathbb{N}}$ (or $\mathcal{M}_{\mathbb{B}, \mathbb{N} \vee}$ ), we put $u_{A}=u_{\mathbf{j}}$ if $A=A(\mathbf{i}, \mathbf{j})$. Now we are in a position to state and prove our main theorem.

\section{Theorem 5.1 There exists a bijection}

$$
\mathcal{M}_{\mathbb{A}, \mathbb{N}} \times \mathcal{M}_{\mathbb{B}, \mathbb{N}^{\vee}} \longrightarrow \mathcal{M}_{\mathbb{A}, \mathbb{B}} \times \mathcal{M}_{\mathbb{B}, \mathbb{N} \vee} \times \mathcal{M}_{\mathbb{A}, \mathbb{N}}
$$

sending $(X, Y)$ to $\left(Z, Y^{\prime}, X^{\prime}\right)$ such that

(1) $\mathrm{wt}_{\mathbb{A}}(X)=\mathrm{wt}_{\mathbb{A}}\left(X^{\prime}\right)+\mathrm{wt}_{\mathbb{A}}(Z)$ and $\mathrm{wt}_{\mathbb{B}}(Y)=\mathrm{wt}_{\mathbb{B}}\left(Y^{\prime}\right)+\mathrm{wt}_{\mathbb{B}}(Z)$,

(2) $u_{X} u_{Y}=t^{|Z|} u_{Y^{\prime}} u_{X^{\prime}}$ where $Z=\left(z_{i j}\right)$ and $|Z|=\sum_{i, j} z_{i j}$.

Proof It is obtained by composing the following bijections, which preserve $\mathrm{wt}_{\mathbb{A}}, \mathrm{wt}_{\mathbb{B}}$ and (4.1): 


$$
\begin{aligned}
& \mathcal{M}_{\mathbb{A}, \mathbb{N}} \times \mathcal{M}_{\mathbb{B}, \mathbb{N} \vee} \\
& \longrightarrow \bigsqcup_{\mu \in \mathcal{P}_{\mathbb{A}}, v \in \mathcal{P}_{\mathbb{B}}} \mathcal{B}_{\mu} \times S S T_{\mathbb{A}}(\mu) \times \mathcal{B}_{v}^{\vee} \times S S T_{\mathbb{B}}(v) \quad \text { by (2.3) and (2.4) } \\
& \longrightarrow \bigsqcup_{\mu \in \mathcal{P}_{\mathbb{A}}, v \in \mathcal{P}_{\mathbb{B}}} S S T_{\mathbb{A}}(\mu) \times S S T_{\mathbb{B}}(\nu) \times \mathcal{B}_{\mu} \times \mathcal{B}_{v}^{\vee} \\
& \longrightarrow \bigsqcup_{\mu \in \mathcal{P}_{\mathbb{A}}, v \in \mathcal{P}_{\mathbb{B}}} S S T_{\mathbb{A}}(\mu) \times S S T_{\mathbb{B}}(\nu) \times\left(\bigsqcup_{\lambda \subset \mu, v} \mathcal{B}_{\nu / \lambda}^{\vee} \times \mathcal{B}_{\mu / \lambda}\right) \quad \text { by (3.3) } \\
& \longrightarrow \bigsqcup_{\mu \in \mathcal{P}_{\mathbb{A}}, v \in \mathcal{P}_{\mathbb{B}}} \bigsqcup_{\lambda \subset \mu, v} S S T_{\mathbb{B}}(\nu) \times \mathcal{B}_{\nu / \lambda}^{\vee} \times S S T_{\mathbb{A}}(\mu) \times \mathcal{B}_{\mu / \lambda} \\
& \longrightarrow \bigsqcup_{\lambda \in \mathcal{P}_{\mathbb{A}} \cap \mathcal{P}_{\mathbb{B}}} S S T_{\mathbb{B}}(\lambda) \times \mathcal{M}_{\mathbb{B}, \mathbb{N} \vee} \times S S T_{\mathbb{A}}(\lambda) \times \mathcal{M}_{\mathbb{A}, \mathbb{N}} \quad \text { by (2.5) and (2.6) } \\
& \longrightarrow\left(\bigsqcup_{\lambda \in \mathcal{P}_{\mathbb{A}} \cap \mathcal{P}_{\mathbb{B}}} S S T_{\mathbb{B}}(\lambda) \times S S T_{\mathbb{A}}(\lambda)\right) \times \mathcal{M}_{\mathbb{B}, \mathbb{N}^{\vee}} \times \mathcal{M}_{\mathbb{A}, \mathbb{N}} \\
& \longrightarrow \mathcal{M}_{\mathbb{A}, \mathbb{B}} \times \mathcal{M}_{\mathbb{B}, \mathbb{N} \vee} \times \mathcal{M}_{\mathbb{A}, \mathbb{N}} \text { by (2.3). }
\end{aligned}
$$

Now, let us consider the non-commutative character identity associated with Theorem 5.1. We first define the plactic Cauchy products

$$
\mathcal{Q}\left(\mathbf{x}_{\mathbb{A}}\right)=\overrightarrow{\prod_{a \in \mathbb{A}}} \mathcal{Q}\left(x_{a}\right), \quad \mathcal{P}\left(\mathbf{x}_{\mathbb{B}}\right)=\overrightarrow{\prod_{b \in \mathbb{B}}} \mathcal{P}\left(x_{b}\right),
$$

where the products are given with respect to the linear ordering on $\mathbb{A}$ or $\mathbb{B}$ so that smaller terms are to the left, and

$$
\begin{aligned}
& \mathcal{Q}\left(x_{a}\right)= \begin{cases}\frac{1}{\cdots\left(1-u_{2} x_{a}\right)\left(1-u_{1} x_{a}\right)}, & \text { if }|a|=0, \\
\left(1+u_{1} x_{a}\right)\left(1+u_{2} x_{a}\right) \cdots, & \text { if }|a|=1,\end{cases} \\
& \mathcal{P}\left(x_{b}\right)= \begin{cases}\frac{1}{\cdots\left(1-u_{2} \vee x_{b}\right)\left(1-u_{1} \vee x_{b}\right)}, & \text { if }|b|=0, \\
\left(1+u_{1} \vee x_{b}\right)\left(1+u_{2} \vee x_{b}\right) \cdots, & \text { if }|b|=1 .\end{cases}
\end{aligned}
$$

We assume that $\mathbf{x}_{\mathbb{A}}$ and $\mathbf{x}_{\mathbb{B}}$ commute with $\mathbf{u}$. Note that

$$
\mathcal{Q}\left(\mathbf{x}_{\mathbb{A}}\right)=\sum_{\lambda \in \mathcal{P}_{\mathbb{A}}} s_{\lambda}(\mathbf{u}) s_{\lambda}\left(\mathbf{x}_{\mathbb{A}}\right), \quad \mathcal{P}\left(\mathbf{x}_{\mathbb{B}}\right)=\sum_{\lambda \in \mathcal{P}_{\mathbb{B}}} s_{\lambda}^{\vee}(\mathbf{u}) s_{\lambda}\left(\mathbf{x}_{\mathbb{B}}\right),
$$

by (2.3) and (2.4).

\section{Corollary 5.2}

$$
\mathcal{Q}\left(\mathbf{x}_{\mathbb{A}}\right) \mathcal{P}\left(\mathbf{x}_{\mathbb{B}}\right)=\frac{\prod_{|a| \neq|b|}\left(1+t x_{a} x_{b}\right)}{\prod_{|a|=|b|}\left(1-t x_{a} x_{b}\right)} \mathcal{P}\left(\mathbf{x}_{\mathbb{B}}\right) \mathcal{Q}\left(\mathbf{x}_{\mathbb{A}}\right) .
$$


Proof By definition, we have

$$
\mathcal{Q}\left(\mathbf{x}_{\mathbb{A}}\right)=\sum_{X \in \mathcal{M}_{\mathbb{A}, \mathbb{N}}} u_{X} \mathbf{x}_{\mathbb{A}}^{\mathrm{wt}_{\mathbb{A}}(X)}, \quad \mathcal{P}\left(\mathbf{x}_{\mathbb{B}}\right)=\sum_{Y \in \mathcal{M}_{\mathbb{B}, \mathbb{N}^{\vee}}} u_{Y} \mathbf{x}_{\mathbb{B}}^{\mathrm{wt}_{\mathbb{B}}(Y)} .
$$

Since the bijections in the proof of Theorem 5.1 preserve the plactic relations (4.1), $w t_{\mathbb{A}}$ and $w t_{\mathbb{B}}$, we obtain the identity.

5.2 Cauchy identity for Schur operators

For $i \in \mathbb{N}$, we define operators $\bar{u}_{i}, \bar{u}_{i} \vee \in \operatorname{End}_{\mathbb{Q}\left[t, t^{-1}\right]}(\Lambda(t))$ by

$$
\begin{aligned}
& \bar{u}_{i \vee}\left(s_{\mu}(\mathbf{x})\right)= \begin{cases}s_{\mu \cup\left\{\left(i, \mu_{i}+1\right)\right\}}(\mathbf{x}), & \text { if } \mu \cup\left\{\left(i, \mu_{i}+1\right)\right\} \in \mathcal{P}, \\
0, & \text { if } \mu \cup\left\{\left(i, \mu_{i}+1\right)\right\} \notin \mathcal{P},\end{cases} \\
& \bar{u}_{i}\left(s_{\mu}(\mathbf{x})\right)= \begin{cases}t s_{\mu \backslash\left\{\left(i, \mu_{i}\right)\right\}}(\mathbf{x}), & \text { if } \mu \backslash\left\{\left(i, \mu_{i}\right)\right\} \in \mathcal{P}, \\
0, & \text { if } \mu \backslash\left\{\left(i, \mu_{i}\right)\right\} \notin \mathcal{P} .\end{cases}
\end{aligned}
$$

These operators are called Schur operators [3]. Let $\overline{\mathcal{U}}(t)$ be the subalgebra of $\operatorname{End}_{\mathbb{Q}\left[t, t^{-1}\right]}(\Lambda(t))$ generated by $\bar{u}_{i}, \bar{u}_{i} \vee(i \in \mathbb{N})$. It is easy to see that there exists a surjective $\mathbb{Q}\left[t, t^{-1}\right]$-algebra homomorphism $\psi: U(t) \rightarrow \bar{U}(t)$ such that $\psi\left(u_{i}\right)=\bar{u}_{i}$ and $\psi\left(u_{i} \vee\right)=\bar{u}_{i} \vee$ for $i \in \mathbb{N}$.

For $\lambda \in \mathcal{P}$, let

$$
s_{\lambda}(\overline{\mathbf{u}})=\sum_{S \in \mathcal{B}_{\lambda}} \bar{u}_{S}, \quad s_{\lambda}^{\vee}(\overline{\mathbf{u}})=\sum_{S \in \mathcal{B}_{\lambda}^{\vee}} \bar{u}_{S},
$$

where $\bar{u}_{S}=\psi\left(u_{S}\right)$ for $S \in \mathcal{B}_{\lambda}$ or $\mathcal{B}_{\lambda}^{\vee}$. For $\lambda, \mu \in \mathcal{P}$, we have

$$
s_{\mu}^{\vee}(\overline{\mathbf{u}})\left(s_{\lambda}(\mathbf{x})\right)=s_{\lambda}(\mathbf{x}) s_{\mu}(\mathbf{x}), \quad s_{\mu}(\overline{\mathbf{u}})\left(s_{\lambda}(\mathbf{x})\right)=t^{|\mu|} s_{\lambda / \mu}(\mathbf{x})
$$

(see [3]). We also have

$$
\begin{aligned}
& \overline{\mathcal{Q}}\left(\mathbf{x}_{\mathbb{A}}\right)=\overrightarrow{\prod_{a \in \mathbb{A}}} \overline{\mathcal{Q}}\left(x_{a}\right)=\sum_{\lambda \in \mathcal{P}_{\mathbb{A}}} s_{\lambda}(\overline{\mathbf{u}}) s_{\lambda}\left(\mathbf{x}_{\mathbb{A}}\right), \\
& \overline{\mathcal{P}}\left(\mathbf{x}_{\mathbb{B}}\right)=\vec{\prod} \overline{\mathcal{P}}\left(x_{b}\right)=\sum_{b \in \mathbb{B}} s_{\lambda}^{\vee}(\overline{\mathbf{u}}) s_{\lambda}\left(\mathbf{x}_{\mathbb{B}}\right),
\end{aligned}
$$

where $\overline{\mathcal{P}}\left(x_{a}\right)$ and $\overline{\mathcal{Q}}\left(x_{b}\right)$ are obtained from $\mathcal{P}\left(x_{a}\right)$ and $\mathcal{Q}\left(x_{b}\right)$ by replacing $u_{i}, u_{i} \vee$ with $\bar{u}_{i}, \bar{u}_{i \vee}$, respectively. Therefore, the products $\overline{\mathcal{Q}}\left(\mathbf{x}_{\mathbb{A}}\right) \overline{\mathcal{P}}\left(\mathbf{x}_{\mathbb{B}}\right)$ and $\overline{\mathcal{P}}\left(\mathbf{x}_{\mathbb{B}}\right) \overline{\mathcal{Q}}\left(\mathbf{x}_{\mathbb{A}}\right)$ are well defined, and the identity in Corollary 5.2 gives the following, which recovers the generalized Cauchy identity for Schur operators [3] when $t=1$ :

$$
\overline{\mathcal{Q}}\left(\mathbf{x}_{\mathbb{A}}\right) \overline{\mathcal{P}}\left(\mathbf{x}_{\mathbb{B}}\right)=\frac{\prod_{|a| \neq|b|}\left(1+t x_{a} x_{b}\right)}{\prod_{|a|=|b|}\left(1-t x_{a} x_{b}\right)} \overline{\mathcal{P}}\left(\mathbf{x}_{\mathbb{B}}\right) \overline{\mathcal{Q}}\left(\mathbf{x}_{\mathbb{A}}\right) .
$$




\subsection{Knuth correspondence for skew tableaux}

Fix $\alpha, \beta \in \mathcal{P}$. For $w=w_{1} \ldots w_{r} \in \mathcal{W}$, we define $\left(S^{(k)}, T^{(k)}\right) \in \mathcal{B}_{\sigma^{(k)}, \tau^{(k)}}(1 \leq$ $k \leq r)$ inductively as follows: (1) $\left(S^{(0)}, T^{(0)}\right)=\left(H_{\alpha}, \emptyset\right),(2)\left(S^{(k)}, T^{(k)}\right)=\left(w_{k} \rightarrow\right.$ $\left.\left(S^{(k-1)}, T^{(k-1)}\right)\right)$ if $w_{k} \in \mathbb{N}$ and $\left(S^{(k)}, T^{(k)}\right)=\left(\left(S^{(k-1)}, T^{(k-1)}\right) \leftarrow w_{k}\right)$ if $w_{k} \in \mathbb{N}^{\vee}$ for $1 \leq k \leq r$.

Let us say that $w$ is a Littlewood-Richardson (simply LR) word of shape $(\alpha, \beta)$ if $\left(S^{(k)}, T^{(k)}\right)=\left(H_{\sigma^{(k)}}, \emptyset\right)$ for $1 \leq k \leq r$, and $\sigma^{(r)}=\beta$. Note that for $1 \leq k \leq r$, $\left|\sigma^{(k)}\right|=\left|\sigma^{(k-1)}\right|+1$ if $w_{k} \in \mathbb{N}$ and $\left|\sigma^{(k)}\right|=\left|\sigma^{(k-1)}\right|-1$ if $w_{k} \in \mathbb{N}^{\vee}$ (we assume that $\left.\sigma^{(0)}=\alpha\right)$. By definition, the subword $w_{s} w_{s+1} \ldots w_{t}$ of $w$ is also an LR word of shape $\left(\sigma^{(s-1)}, \sigma^{(t)}\right)$ for $1 \leq s<t \leq r$.

Lemma 5.3 For $w \in \mathcal{W}, w$ is an LR word of shape $(\alpha, \beta)$ if and only if $H_{\alpha} \otimes w \equiv H_{\beta}$. In particular, if $w$ is an LR word of shape $(\alpha, \beta)$ and $w^{\prime} \equiv w$ for $w^{\prime} \in \mathcal{W}$, then $w^{\prime}$ is also an LR word of shape $(\alpha, \beta)$.

Proof We keep the above notations. Suppose that $w$ is an LR word of shape $(\alpha, \beta)$. Since $H_{\alpha} \otimes w_{1} \ldots w_{k} \equiv\left(H_{\sigma^{(k)}}, \emptyset\right) \equiv H_{\sigma^{(k)}}$ for $1 \leq k \leq r$, we have $H_{\alpha} \otimes w \equiv H_{\beta}$.

Conversely, suppose that $H_{\alpha} \otimes w \equiv H_{\beta} \equiv\left(H_{\beta}, \emptyset\right)$. If $\tau^{(k)} \neq \emptyset$ (that is, $T^{(k)} \neq \emptyset$ ) for some $k$, then we have $\tau^{(r)} \neq \varnothing$ (that is, $T^{(r)} \neq \emptyset$ ) by definition of the insertions (Sect. 3.1), which contradicts the fact that $\left(S^{(r)}, T^{(r)}\right) \equiv H_{\alpha} \otimes w \equiv\left(H_{\beta}, \emptyset\right)$. Hence $\tau^{(k)}=\emptyset$ (that is, $T^{(k)}=\emptyset$ ) for $1 \leq k \leq r$ and $\sigma^{(r)}=\beta$.

Now suppose that $S^{(k)} \neq H_{\sigma^{(k)}}$ for some $1 \leq k<r$, which is equivalent to saying that $\widetilde{e}_{i} S^{(k)} \neq \mathbf{0}$ for some $i \geq 1$. Then

$$
\widetilde{e}_{i} H_{\beta} \equiv \widetilde{e}_{i}\left(H_{\alpha} \otimes w\right) \equiv \widetilde{e}_{i}\left(S^{(k)} \otimes w_{k+1} \ldots w_{r}\right)=\left(\widetilde{e}_{i} S^{(k)}\right) \otimes w_{k+1} \ldots w_{r} \neq \mathbf{0},
$$

which is also a contradiction. Hence $w$ is an LR word of shape $(\alpha, \beta)$.

For $\lambda, \mu \in \mathcal{P}$ with $|\lambda|=|\alpha|+|\mu|$, we have by (2.1)

$$
\left\{S \in \mathcal{B}_{\mu} \mid w(S) \text { is an LR word of shape }(\alpha, \lambda)\right\} \stackrel{1-1}{\longleftrightarrow} \mathbf{L R}_{\alpha \mu}^{\lambda} .
$$

For $\lambda, \nu \in \mathcal{P}$ with $|\lambda|=|\beta|+|\nu|$, we have by (3.2)

$$
\left\{S \in \mathcal{B}_{v}^{\vee} \mid w(S) \text { is an LR word of shape }(\lambda, \beta)\right\} \stackrel{1-1}{\longleftrightarrow} \mathbf{L R}_{\beta \nu}^{\lambda} \text {. }
$$

Let $\left(\mathcal{B}_{\mu} \times \mathcal{B}_{v}^{\vee}\right)_{(\alpha, \beta)}$ be the set of $(S, T) \in \mathcal{B}_{\mu} \times \mathcal{B}_{v}^{\vee}$ such that $w(S) w(T)$ is an LR word of shape $(\alpha, \beta)$. Combining (5.1) and (5.2), we have

$$
\left(\mathcal{B}_{\mu} \times \mathcal{B}_{v}^{\vee}\right)_{(\alpha, \beta)} \stackrel{1-1}{\longleftrightarrow} \bigsqcup_{\lambda} \mathbf{L R}_{\alpha \mu}^{\lambda} \times \mathbf{L} \mathbf{R}_{\beta \nu}^{\lambda} .
$$

Similarly, for $\sigma, \tau \in \mathcal{P}$, let $\left(\mathcal{B}_{\tau}^{\vee} \times \mathcal{B}_{\sigma}\right)_{(\alpha, \beta)}$ be the set of $(S, T) \in \mathcal{B}_{\tau}^{\vee} \times \mathcal{B}_{\sigma}$ such that $w(S) w(T)$ is an LR word of shape $(\alpha, \beta)$. As in (5.3), we have a bijection

$$
\left(\mathcal{B}_{\tau}^{\vee} \times \mathcal{B}_{\sigma}\right)_{(\alpha, \beta)} \stackrel{1-1}{\longleftrightarrow} \bigsqcup_{\lambda} \mathbf{L R}_{\lambda \tau}^{\alpha} \times \mathbf{L} \mathbf{R}_{\lambda \sigma}^{\beta} .
$$


Corollary 5.4 Let $\alpha, \beta, \mu, v \in \mathcal{P}$ be given. The bijection (3.2) when restricted to $\left(\mathcal{B}_{\mu} \times \mathcal{B}_{v}^{\vee}\right)_{(\alpha, \beta)}$ gives the following bijection:

$$
\bigsqcup_{\lambda} \mathbf{L R}_{\alpha \mu}^{\lambda} \times \mathbf{L} \mathbf{R}_{\beta \nu}^{\lambda} \longrightarrow \bigsqcup_{\eta, \zeta, \sigma, \tau} \mathbf{L R}_{\eta \tau}^{\alpha} \times \mathbf{L} \mathbf{R}_{\eta \sigma}^{\beta} \times \mathbf{L} \mathbf{R}_{\sigma \zeta}^{\mu} \times \mathbf{L} \mathbf{R}_{\tau \zeta}^{v}
$$

Proof Since the bijection (3.2) preserves the plactic relations or the crystal equivalence, we have by Lemma 5.3

$$
\left(\mathcal{B}_{\mu} \times \mathcal{B}_{\nu}^{\vee}\right)_{(\alpha, \beta)} \longrightarrow \bigsqcup_{\zeta, \sigma, \tau}\left(\mathcal{B}_{\tau}^{\vee} \times \mathcal{B}_{\sigma}\right)_{(\alpha, \beta)} \times \mathbf{L} \mathbf{R}_{\sigma \zeta}^{\mu} \times \mathbf{L} \mathbf{R}_{\tau \zeta}^{\nu}
$$

Hence, it follows from (5.3) and (5.4).

Let $\left(\mathcal{M}_{\mathbb{A}, \mathbb{N}} \times \mathcal{M}_{\mathbb{B}, \mathbb{N} \vee}\right)_{(\alpha, \beta)}$ be the set of $\left(A, A^{\prime}\right)$ such that $\mathbf{j} \cdot \mathbf{j}^{\prime} \in \mathcal{W}$ is an LR word of shape $(\alpha, \beta)$, where $A=A(\mathbf{i}, \mathbf{j})$ and $A^{\prime}=A\left(\mathbf{i}^{\prime}, \mathbf{j}^{\prime}\right)$, and let $\left(\mathcal{M}_{\mathbb{B}, \mathbb{N}} \vee \times \mathcal{M}_{\mathbb{A}, \mathbb{N}}\right)_{(\alpha, \beta)}$ be defined in the same way.

Now, we recover the Knuth type correspondence for skew tableaux by Sagan and Stanley [17] as a restriction of the bijection in Theorem 5.1 to the set of LR words of shape $(\alpha, \beta)$.

Theorem 5.5 Let $\alpha, \beta \in \mathcal{P}$ be given. The bijection in Theorem 5.1 when restricted to $\left(\mathcal{M}_{\mathbb{A}, \mathbb{N}} \times \mathcal{M}_{\mathbb{B}, \mathbb{N} \vee}\right)_{(\alpha, \beta)}$ gives a bijection

$$
\bigsqcup_{\lambda} S S T_{\mathbb{A}}(\lambda / \alpha) \times S S T_{\mathbb{B}}(\lambda / \beta) \longrightarrow \bigsqcup_{\eta} \mathcal{M}_{\mathbb{A}, \mathbb{B}} \times S S T_{\mathbb{A}}(\beta / \eta) \times S S T_{\mathbb{B}}(\alpha / \eta) .
$$

Proof Since the bijection in Theorem 5.1 preserves the plactic relations, we have a bijection by Lemma 5.3

$$
\left(\mathcal{M}_{\mathbb{A}, \mathbb{N}} \times \mathcal{M}_{\mathbb{B}, \mathbb{N}} \vee\right)_{(\alpha, \beta)} \longrightarrow \mathcal{M}_{\mathbb{A}, \mathbb{B}} \times\left(\mathcal{M}_{\mathbb{B}, \mathbb{N}} \vee \times \mathcal{M}_{\mathbb{A}, \mathbb{N}}\right)_{(\alpha, \beta)} .
$$

On the other hand, we have

$$
\begin{aligned}
& \left(\mathcal{M}_{\mathbb{A}, \mathbb{N}} \times \mathcal{M}_{\mathbb{B}, \mathbb{N} \vee}\right)_{(\alpha, \beta)} \\
& \stackrel{1-1}{\longleftrightarrow} \bigsqcup_{\mu \in \mathcal{P}_{\mathbb{A}}, v \in \mathcal{P}_{\mathbb{B}}}\left(\mathcal{B}_{\mu} \times \mathcal{B}_{\nu}^{\vee}\right)_{(\alpha, \beta)} \times S S T_{\mathbb{A}}(\mu) \times S S T_{\mathbb{B}}(\nu) \\
& \stackrel{1-1}{\longleftrightarrow} \bigsqcup_{\substack{\mu \in \mathcal{P}_{\mathbb{A}}, \nu \in \mathcal{P}_{\mathbb{B}} \\
\mu, \nu \subset \lambda}} \mathbf{L R _ { \alpha \mu } ^ { \lambda }} \times \mathbf{L R}_{\beta \nu}^{\lambda} \times S S T_{\mathbb{A}}(\mu) \times S S T_{\mathbb{B}}(\nu) \quad \text { by }(5.3) \\
& \stackrel{1-1}{\longleftrightarrow} \bigsqcup_{\substack{\mu \in \mathcal{P}_{\mathbb{A}}, v \in \mathcal{P}_{\mathbb{B}} \\
\mu, \nu \subset \lambda}} S S T_{\mathbb{A}}(\mu) \times \mathbf{L R}_{\mu \alpha}^{\lambda} \times S S T_{\mathbb{B}}(\nu) \times \mathbf{L R}_{\nu \beta}^{\lambda} \\
& \stackrel{1-1}{\longleftrightarrow} \bigsqcup_{\alpha, \beta \subset \lambda} S S T_{\mathbb{A}}(\lambda / \alpha) \times S S T_{\mathbb{B}}(\lambda / \beta) \quad \text { by (2.2). }
\end{aligned}
$$


Similarly, we have

$$
\begin{aligned}
& \left(\mathcal{M}_{\mathbb{B}, \mathbb{N} \vee} \times \mathcal{M}_{\mathbb{A}, \mathbb{N}}\right)_{(\alpha, \beta)} \\
& \stackrel{1-1}{\longleftrightarrow} \bigsqcup_{\sigma \in \mathcal{P}_{\mathbb{A}}, \tau \in \mathcal{P}_{\mathbb{B}}}\left(\mathcal{B}_{\tau}^{\vee} \times \mathcal{B}_{\sigma}\right)_{(\alpha, \beta)} \times S S T_{\mathbb{A}}(\sigma) \times S S T_{\mathbb{B}}(\tau) \\
& \stackrel{1-1}{\longleftrightarrow} \bigsqcup_{\substack{\sigma \in \mathcal{P}_{\mathbb{A}}, \tau \in \mathcal{P}_{\mathbb{B}} \\
\eta \subset \alpha, \beta}} \mathbf{L R}_{\eta \tau}^{\alpha} \times \mathbf{L R}_{\eta \sigma}^{\beta} \times S S T_{\mathbb{A}}(\sigma) \times S S T_{\mathbb{B}}(\tau) \quad \text { by (5.4) } \\
& \stackrel{1-1}{\longleftrightarrow} \bigsqcup_{\substack{\sigma \in \mathcal{P}_{\mathbb{A}}, \tau \in \mathcal{P}_{\mathbb{B}} \\
\eta \subset \alpha, \beta}} S S T_{\mathbb{A}}(\sigma) \times \mathbf{L R}_{\sigma \eta}^{\beta} \times S S T_{\mathbb{B}}(\tau) \times \mathbf{L R}_{\tau \eta}^{\alpha} \\
& \stackrel{1-1}{\longleftrightarrow} \bigsqcup_{\eta \subset \alpha, \beta} S S T_{\mathbb{A}}(\beta / \eta) \times S S T_{\mathbb{B}}(\alpha / \eta) \quad \text { by (2.2). }
\end{aligned}
$$

Combining with (5.5), we obtain the result.

Acknowledgement The author would like to thank the referees for careful reading of the manuscript and helpful comments on it.

\section{References}

1. Benkart, G., Sottile, F., Stroomer, J.: Tableau switching: algorithms and applications. J. Comb. Theory, Ser. A 76, 11-43 (1996)

2. Berele, A., Regev, A.: Hook Young diagrams with applications to combinatorics and to representations of Lie superalgebras. Adv. Math. 64, 118-175 (1987)

3. Fomin, S.: Schur operators and Knuth correspondences. J. Comb. Theory, Ser. A 72, 277-292 (1995)

4. Fomin, S., Greene, C.: Noncommutative Schur functions and their applications. Discrete Math. 193(13), 179-200 (1998)

5. Frenkel, I.B.: Representations of Kac-Moody algebras and dual resonance models. In: Applications of Group Theory in Physics and Mathematical Physics. Lectures in Appl. Math., vol. 21, pp. 325-353. AMS, Providence (1985)

6. Fulton, W.: Young Tableaux. London Mathematical Society Student Texts, vol. 35. Cambridge University Press, Cambridge (1997)

7. Kashiwara, M.: On crystal bases. In: Representations of Groups. CMS Conf. Proc., vol. 16, pp. 155197. Amer. Math. Soc., Providence (1995)

8. Kashiwara, M.: Crystal bases of modified quantized enveloping algebra. Duke Math. J. 73, 383-413 (1994)

9. Kashiwara, M., Nakashima, T.: Crystal graphs for representations of the $q$-analogue of classical Lie algebras. J. Algebra 165, 295-345 (1994)

10. Knuth, D.: Permutations, matrices, and the generalized Young tableaux. Pac. J. Math. 34, 709-727 (1970)

11. Kwon, J.-H.: Differential operators and crystals of extremal weight modules. Adv. Math. 222, 13391369 (2009)

12. Kwon, J.-H.: Crystal bases of modified quantized enveloping algebras and a double RSK correspondence. Preprint (2010), arXiv:1002.1509. J. Comb. Theory Ser. A (to appear)

13. Lam, T.: Ribbon Schur operators. Eur. J. Comb. 29(1), 343-359 (2008)

14. Lascoux, A., Schützenberger, M.P.: Le monoïde plaxique. In: Noncommutative Structures in Algebra and Geometric Combinatorics, Naples, 1978. Quad. "Ricerca Sci.", vol. 109, pp. 129-156. CNR, Rome (1981) 
15. Littelmann, P.: A plactic algebra for semisimple Lie algebras. Adv. Math. 124, 312-331 (1996)

16. Macdonald, I.G.: Symmetric Functions and Hall Polynomials, 2nd edn. Oxford University Press, Oxford (1995)

17. Sagan, B.E., Stanley, R.: Robinson-Schensted algorithms for skew tableaux. J. Comb. Theory, Ser. A 55, 161-193 (1990)

18. Stembridge, J.R.: Rational tableaux and the tensor algebra of $\mathfrak{g l}_{n}$. J. Comb. Theory, Ser. A 46, 79-120 (1987)

19. Stroomer, J.: Insertion and the multiplication of rational Schur functions. J. Comb. Theory, Ser. A 65 , 79-116 (1994)

20. Thomas, G.P.: On Schensted's construction and the multiplication of Schur functions. Adv. Math. 30, 8-32 (1978) 